EXEMPLARIa Classica

Journal of Classical Philology

16, 2012 , pp. $45-73$

ISSN 1699-3225

\title{
UNA POSSIBILE RILETTURA DEI CARMI 113 E 94 DI CATULLO SULLE TRACCE DI UN CICLO DI MUCIA
}

\author{
Alex Agnesini \\ Università degli Studi di Parma \\ alex.agnesini@unipr.it
}

SuMMARY

This paper's aim is to support, firstly, the identification of Mucia in Catull. 113.2, by means of a wordplay based on $\mu$ orx $\eta^{\prime}$ and $\chi^{i} \lambda \lambda_{1 \alpha}$, and, secondly, Baehrens' emendation Moechula in Catull. 94.1 instead of the second instance of mentula. This reading would shed light on some problems of both poems.

KEYWORDS

Catullus, textual criticism, wordplay

\section{RESUMEN}

La presenza di Mucia nei carmi 113 e 94 potrebbe dare luce a problemi ancora non risolti. Nel 113 si giustifica la sua presenza attraverso un gioco di parole con $\mu$ orxí e $X^{i} \lambda_{\imath} \mathbf{\alpha}$ e nel 94, riprendendo la proposta di Baehrens di leggere al v.1 Moechula. Questa lettura potrebbe far luce su alcuni problemi di entrambi i poemi.

Palabras Clave

Catullo, critica del testo, lusus verbale

Fecha de recepción: 21/03/2012

Fecha de aceptación y versión final: 23/07/2012

Il carme 113 di Catullo non sembra presentare particolari problemi testuali. Antonio Ramírez de Verger ${ }^{1}$, ultimo editore di Catullo, pubblica così il carme:

Consule Pompeio primum duo, Cinna, solebant Maeciliam: facto consule nunc iterum manserunt duo, sed creuerunt milia in unum singula: fecundum semen adulterio!

Laddove anche la tradizione manoscritta non sia affetta da insormontabili guasti, sovente in Catullo si registra la difficoltà di intendere il testo. Nel

${ }^{1}$ C. Valerii Catulli Carmina - Catulo Poemas, edición, traducción y comentario, Ana Pérez Vega - A. Ramírez de Verger, Huelva 2005. 
caso specifico del carme 113, i manoscritti antiquiori rinunciavano a dividere la serie continua dei carmi e solo alla metà del ' $400^{2}$ si registrano le prime attestazioni della separazione dei due distici che potevano essere distinti dai testi contigui, contro un certo Naso (Catull. 112) e contro Mentula (carme 114, quello del saltus Firmanus), senza una minuziosa comprensione della pericope. Per quanto riguarda i titoli ${ }^{3}$, la tradizione dà rilievo al dedicatario, Cinna ${ }^{4}$ : solo due, peraltro testimoniati una sola volta ${ }^{5}$, sembrano cogliere l'aspetto saliente del carme nel riferimento ai consolati di Pompeo (Tempore Pompei e In Pompeium).

Per quanto riguarda il testo, l'unico vero guasto da segnalare è al v. 4 , ove la stragrande maggioranza dei codici legge singulum, che determina un'impossibile sequenza cretica ${ }^{6}$. Anche per questa menda bisogna aspettare la metà del secolo $\mathrm{XV}^{7}$ per avere una lettura metrica corretta, singula ${ }^{8}$, che è quella oggidì accolta.

${ }^{2}$ Il primo è San Lorenzo del Escorial, Real Biblioteca del Escorial, Ç IV 22(b), risalente alla metà del secolo, poi a partire dagli anni ' 60 del XV secolo la separazione sarà piuttosto vulgata. Infatti più della metà dei manoscritti e delle edizioni a stampa tra 1460 (circa) e 1520 (circa) isolano i due distici costituenti Catull. 113 dai carmi precedente e successivo.

${ }^{3}$ Non si intende prendere posizione circa un'eventuale genuinità dei titoli che si possono reperire nei manoscritti catulliani. Essi possono costituire una testimonianza in merito alla comprensione del testo tràdito.

${ }^{4}$ Le prime attestazioni sono De Cinna, in seguito verrà preferito Ad Cinnam.

${ }^{5}$ Rispettivamente da Pesaro, Biblioteca Oliveriana, 1167 e Stato della Città del Vaticano, Biblioteca Vaticana, Urb. lat. 812.

${ }^{6}$ Lo Scaligero intende salvare la lezione manoscritta singulum: «Liber habebat Singulum, non Singula. ita exteritur media, ut in Aurunculeia. Plautus Clitellaria [sic]: Atat, singulum video vestigium [Cist.701-701a Stockert]》 (Catulli, Tibulli, Properti, nova editio. I. Scaliger Iul. Caesaris f. recensuit. Eiusdem in eosdem Castigationum Liber, Lutetiae 1577, 106). Ma Aurunculeia in Catull. 61 non necessita di sincope se si accoglie la colometria comunemente diffusa ai vv. 82-3 (flere desine: non tibi Au- / runculeia periculum est), invece di quella scelta dallo Scaligero (flere desine. non tibi / Aurunculeia periculum est). Per quanto riguarda il passo plautino i recenti editori, sulla scorta di F. Skutsch, Kleine Schriften, Leipzig 1914, 246 n. 2, rinunciano a difendere l'ordo tràdito, come lo Scaligero, e invertendo l'ordine delle parole stampano attat, / singulum uestigium uideo (Plaut. Cist. 701-701a Stockert=Lindsay).

${ }^{7}$ I testimoni più antichi, stando alla datazione di D. F. S. Thomson, Catullus. Edited with a textual and interpretative commentary by D. F. S. T., Toronto 1997, sarebbero ancora Esc. Ç IV 22(b), poi Stato della Città del Vaticano, Biblioteca Apostolica, Pal. lat. 1652 («prob. ca. 1455»), Oxford, Bodleian Library, e.15 (posteriore al 1459). Si noti che gli ultimi due manoscritti potrebbero essere stati influenzati dal magistero di Pietro Odo (cf. Thomson, Catullus, $81 \mathrm{n}$. 70 e 85 n. 104) e il Palatino venne approntato da Giannozzo Manetti: tuttavia, dal momento che i due manoscritti presentano il carme 113 legato a quanto viene prima senza soluzione di continuità e che al v. 2 bisogna intendere la prima parola come ablativo per salvare il metro, davvero poco si può ipotizzare circa l'esegesi del carme. Primo ad attestarla è ancora Esc. Ç IV 22(b), tuttavia la lezione singulum avrà ancora diffusione: solo a partire dall'editio princeps.

${ }^{8}$ Per quanto ci risulta, oltre a singula, la tradizione restituisce un solo altro tentativo di sanare il metro. Posteriore probabilmente al 1472 è la lezione di London, British Library, Add. 11674 che, in maniera paragonabile a quella dello Scaligero (cf. supra), legge singlum: tale sincope, diffusa maggiormente in periodo tardo antico e medievale, non è impossibile ai tempi di Catullo (cf. Lucr. 6.1067 quae memorare queam inter se singlariter apta). Con questa 
Infine altre oscillazioni nella tradizione degne di nota sono al v. 2: infatti Maeciliam ha un pendant in G, che legge mecili $\bar{a}^{9}$, mentre gran parte della tradizione reca mecilia o evidenti corruttele di essa (cf. infra). Conformemente alla stragrande maggioranza dei recenziori, che non divergono dalla desinenza di mecilia di O R, l'editio princeps e la Romana stampano mecilia, forse intendendolo ablativo, unico modo per rispettare il metro ${ }^{10}$. Tuttavia non abbiamo certezze sull'esegesi quattrocentesca del lemma (per lo meno fino al 1481, cf. infra): infatti sono attestate, in luogo di mecilia, medii $^{11}$, militia $^{12}$, cecilia $^{13}$ e addirittura alcuni editori (le edizioni di Parma e di Reggio Emilia) dividono la parola in un inconsulto me cilia, che dovrebbe concordare con duo solebant ${ }^{14}$.

lettura, al v. 4 si avrebbe sing $(u)$ lum fecundum semen adulterio: se da una parte si ha il vantaggio di evitare la ridondanza di milia in unum singula, dall'altra si è costretti ad assegnare due attributi per semen, collegati asindeticamente, perdendo anche univocità di interpretazione. Infatti il fulmen così ricostruito potrebbe significare sia che l'adulterio ha un solo fecondo seme, sia che questo adulterio ha un seme singolarmente fecondo.

${ }^{9}$ Tale lezione è presente altrove solo nei manoscritti imparentati S. Daniele del Friuli, Biblioteca Guarneriana, 56; San Lorenzo del Escorial, Real Biblioteca del Escorial, Ç IV 22(a); Napoli, Biblioteca Nazionale, IV F 21. Infatti secondo A. Ghiselli (a cura di), Catullo. Guarnerianus 56. Escorialensis ç IV 22, Bologna 1987, 35 è probabile che Ç IV 22(a) derivi dal guarneriano e Thomson, Catullus, 80 n. 65 definisce il manoscritto napoletano IV F 21 «copy» di Ç IV 22(a).

${ }^{10} \mathrm{~A}$ meno che, come suggerisce un referee, mecilia potesse essere giustificato metricamente dai lettori quattrocenteschi di Catullo con un allungamento in arsi. Tuttavia già con l'edizione vicentina del 1481 per poter leggere moechilia quale neutro plurale si è costretti a integrare at tra moechilia e facto. Dal momento che Partenio (già nell'edizione del 1485, cf. infra $\mathrm{n}$. 26) accoglie tale integrazione, quanto ipotizza il medesimo referee è palesamente inaccettabile: «Parthenius puts a neuter nominative plural in $-a$ here, with the final syllable apparently lengthened in arsi».

${ }^{11}$ Padova, Biblioteca Capitolare, C77, che leggeva con difficoltà il carme, scrivendo, consule pompeio primum duo cinna [il ductus di ci- è molto unito, tanto che la lettura potrebbe dirsi onna] solebat / medii facto consule non iterum / manserunt duo sed creuerunt milia in unum / singulum fecundum se in [inde?] adulterio.

${ }^{12}$ Oliv. 1167 , che presenta un guasto e poco perspicuo consule pompeio primum duo cinna solebat / militia facto consule non iterum / manserunt duo sed creuerunt milia in unum / singulum fecundum semen adulterio (l'amanuense dà al componimento il titolo "Tempore pompei").

${ }^{13}$ Così Milano, Biblioteca Ambrosiana, D 24 sup. Si noti che I. Vossius, Cajus Valerius Catullus et in eum I. Vossii observationes, Londini 1684, 340 testimonia anche l'esistenza della lezione Caecilio, che sarebbe preferibile alla lezione vulgata: «In compluribus scriptum inveni Mechilia, unde demum variae istae prodierunt coniecturae. Sed vero bene se habet scriptura optimi exemplaris; Pompejus primum consul anno urbis DCLXXXIV. una cum Licinio Crasso. Sequens vero annus consules habuit Caecilium Metellum Creticum \& Q. Hortensium. Clarum itaque quid velit Catullus. Hujus Caecili supra quoque meminit in Epigrammate ad Januam. Plures autem fuisse illo tempore Quintos Caecilios Metellos testatur Asconius Paedianus in praefatione orationis pro Cornelio. Quem autem praecipue perstringit Catullus, is videtur fuisse Q. Caecilius Metellus Nepos ,quem malum fuisse civem \& Ciceronis inimicum idem docet Asconius".

${ }^{14}$ Tale scansione in realtà è già presente in due manoscritti degli anni '60 del XV: London, British Library, Add. 11915 (a. 1460 come si evince dalla soscrizione al f. 68v.) e Milano, 
Con l'esegesi della prima parola del v. 2 si tocca l'unico problema tuttora aperto: accolta unanimemente la lezione con l'accusativo singolare, restano dubbi sulla corretta grafia della parola e soprattutto sull'identificazione del personaggio femminile ${ }^{15}$. Infatti la critica catulliana si divide tra chi ${ }^{16}$ nel v. 2 riconosce un'allusione a Mucia, terza moglie di Pompeo, e $\mathrm{chi}^{17}$ ritiene il personaggio citato un membro femminile, altrimenti ignoto, della gens Maecilia. Chi segue quest'ultima esegesi accoglie ovviamente la grafia Maecilia, mentre l'altra parte della critica si divide ulteriormente tra chi concorda con questa grafia e chi propende per altre forme. Rifacendosi, e.g., all'apparato di Ramírez de Verger, si possono citare, oltre all'accolto Maeciliam ${ }^{18}$, anche le proposte Mucillam, Moecillam e Moeciliam.

In effetti la presenza di una Maecilia elimina i problemi di grafia connessi con l'identificazione di Mucia: così Thomson preferisce ipotizzare la presenza di un personaggio sconosciuto (peraltro caso non isolato, in Catullo), recante però un nome attestato nella latinità, piuttosto che giustificare Maecilia quale diminutivo, apparentemente alterato se non corrotto, di Mucia ${ }^{19}$. Inoltre i sostenitori di Mecilia credono che un attacco a Pompeo per mezzo di Mucia sarebbe poco comprensibile perché il carme è databile nel 55 a.C. (cf. v. 2 facto consule nunc iterum ${ }^{20}$. Così si è costretti a credere che tra il $70 \mathrm{e}$

Biblioteca Ambrosiana, H 46 sup. (Thomson, Catullus, 79: «ca. 1460-70»)

${ }^{15} \mathrm{Si}$ noti già la scarsa chiarezza della posizione di R. Ellis, A commentary on Catullus, Oxford $1889^{2}, 495$ che, parlando nel suo commento di Maecilia, afferma: «Pleitner [...] has identified the Maecilia of this epigram with Mucia, wife of Pompeius. The arguments in favor of this view are -1[...] The name Maecilia is a real one, e.g. C. I. L. ii. 1277 Maeciliae P. F. Herennianae: and this throws some slight additional doubt on a theory certainly very plausible».

${ }^{16}$ Cf. e.g., tra gli editori di Catullo, G. Lafaye, Catulle. Poésies, texte établi et traduit par G. F., Paris 1923, 119, che accoglie Mucillam, G. P. Goold (ed. by), Catullus, edited with Introduction, Translation and Notes, second Edition (corrected), London 1989, 264, che legge Mucillam, F. Della Corte (a c. di), Catullo. Le poesie, Milano 1990, 361, che legge Moeciliam, Ramírez de Verger, C. Valerii Catulli Carmina.

${ }^{17}$ Cf. W. Kroll (hrsg. u. erkl. von), C. Valerius Catullus, Stuttgart 19685, 285, Thomson, Catullus, 550, e, più recentemente, e.g., M. B. Skinner, Catullus in Verona: a reading of the elegiac libellus, poems 65-116, Columbus 2003, 138 e 222 n. 31 e C. Hartz, Catulls Epigramme im Kontext hellenistischer Dichtung, Berlin - New York 2007, 162.

${ }^{18}$ Lo studioso, come peraltro altri, adotta tale grafia, intendendo il nome quale allusione a Mucia, cf. Ramírez de Verger, C. Valerii Catulli Carmina, 648.

19 "to claim that a corrupt text accidentally coincided with this form [scil. Maecilia], and then to suppose that what underlies it is Mucilla [...], and further to explain that Mucilla (otherwise unattested) is a diminutive (but why use a diminutive here?) of Mucia, the name of Pompey's third wife, divorced in $62 \mathrm{BC}$ - seven years before the date of this poem - strains credulity» (Thomson, Catullus, 550).

${ }^{20}$ Cf. supra n. 19 Thomson, Catullus e cf. già C.J. Fordyce, Catullus, a commentary, Oxford 1978, 400: «Maecilia is unknown, but the name is a well authenticated gentile name. Pleitner's Mucillam is meant to be a disparaging reference to Pompey's third wife Mucia [...], whom he divorced in 62 b.C. [...]. That was a notorious scandal at the time [...], but when this poem was written, it was an old story [...]. Pompey's repeated consulship is enough in itself to give the epigram its point». 
il 55 a.C. (primo e secondo consolato di Pompeo) Catullo abbia assistito alle gesta di Mecilia, grazie ai cui favori un consistente numero di uomini sono diventati adulteri. Nessun'altra fonte testimonia l' "attività" della donna, ma, se l'esegesi cogliesse nel segno, spettatore interessato doveva essere Cinna, dedicatario del carme e, forse, anche Pompeo, visto che viene utilizzato per delimitare l'arco temporale di tale attività.

Al contrario, la presenza di Mucia nel carme 113 sembra poggiare esclusivamente sul contenuto del carme. La menzione di Pompeo in un contesto adulterino può indurre a credere che la donna citata sia proprio Mucia, ripudiata appunto per infedeltà coniugale ${ }^{21}$. Tale esegesi costringe a credere in una voluta e poco perspicua alterazione di Mucilla oppure a emendare in Mucilla, lemma tuttavia non attestato altrove. Infatti Maecilia, Moecilia, da intendersi allusioni a Mucia, pur concordando in un finale (-ilia) coi manoscritti antiquiori, non trovano un esatto pendant in suffissi né latini né greci ${ }^{22}$.

Inoltre la presenza di Mucia renderebbe giustificabile l'Anrede a Cinna, dacché comunemente Elvio Cinna è identificato con uno sventurato amico di Cesare $^{23}$ : in quanto tale non avrebbe dovuto dispiacergli «una battuta satirica sulle corna di Pompeo che [...] dovette ripudiare Mucia, perché lo tradiva proprio con Cesare» ${ }^{24}$.

Quindi la questione è di fatto aperta e con essa anche la fissazione del testo.

Può giovare fare un passo indietro per vedere se la prima parola del v. 2 è sempre stata intesa in questi due modi. Come abbiamo detto (cf. supra) fino al periodo delle primissime edizioni a stampa di Catullo è difficile capire come si intendesse mecilia (o quanto altro mai si leggesse), tuttavia a partire dall'edizione curata da Giovanni Planza de' Ruffinoni ${ }^{25}$ si può identificare un tentativo

${ }^{21}$ Così pensa, e.g., M. Lenchantin-De Gubernatis (a c. di), Il libro di Catullo, introduzione testo e note, Torino $1933^{3}$, 268: «la citazione dei due consolati di Pompeo non avrebbe ragione di essere se egli non avesse rapporto con le cose a cui C. allude».

${ }^{22}$ In effetti sembra piuttosto difficile credere che Maecilia, nome di per sé attestato, possa costituire un'allusione a Mucia. Forse il finale di parola può avere una qualche corrispondenza

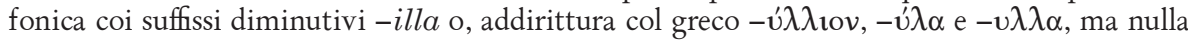
di più.

${ }^{23}$ Ossia il tribuno ucciso dalla folla che lo aveva scambiato col Cinna cesaricida, cf. e.g. F. Della Corte, Personaggi catulliani, Firenze $1976^{2}$, 249. Altri ha ipotizzato che l'Anrede a Cinna si giustifichi in quanto il poeta prima della composizione del carme era passato dalla fazione pompeiana a quella cesariana (cf. C.L. Neudling, A prosopograhpy to Catullus, Oxford 1955, 79, il quale mostra un qualche interesse per tale suggestione). Per una panoramica delle obiezioni a tale identificazione si rimanda a E. Courtney, The Fragmentary Latin Poets, Oxford 2003, 212.

${ }^{24}$ Della Corte, Personaggi, 252 n. 6.

${ }^{25}$ J. Calphurnius, Catulli, Tibulli, propertii Carmina et Statii Syluae, Vincentiae 1481. Se la datazione di Thomson, Catullus, 78 coglie nel segno, «(prob.) 1474», tale esegesi potrebbe già essere attestata in London, British Library, Additional 10386, che legge così: consule Pompeio primum duo Cinna sedebant / mecilia facto Consule nunc iterum / manserunt duo sed creuerunt milia in unum / singula foecundum semen adulterio (titolo del carme: "De 
di intendere la prima parola del v. 2: l'edizione vicentina stampa consule pompeio primum duo cinna sedebant / moechilia at facto consule nunc iterum etc. Quello che ci importa rilevare ora è che moechilia fosse inteso come neutro plurale, concordato con duo e soggetto di sedebant, come Partenio conferma: «męchalia. męche paruę: est autem gręcum diminutiuum tò $\mu$ or $\chi \alpha$ $\lambda^{\prime}$ เov ut gly-

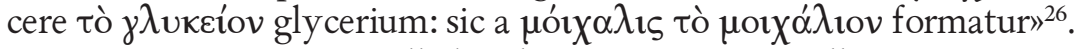

Si giunge poi a Palladio che stampa così Catull. 113:

\section{Ad Cinnam.}

Consule pompeio primum duo cinna sedebant

Moechilia: at facto consule nunc iterum

Manserunt duo: sed creuerunt chilia in unum

Singula fecundum semen adulterio.

L'esegesi è la seguente: «scribit poeta hoc tetrasticon ad cinnam de mirando romae moecharum incremento. Pompeio .n. primum consule duae tantum moechae in urbe sedebant: sed postea cum idem post peius factus esset iterum consul creuerat ad duo milia. [...] moechilium graecum est uocabulum quod parua moecham significat» ${ }^{27}$. Si noti che a testo il Patavino accoglie l'emendamento chilia in luogo di milia, attribuito a un Giovanni Fosforo ${ }^{28}$.

Cinna"). Il codice infatti è il primo (edd. a stampa comprese) a recare sedebant. I dubbi circa la consapevolezza di tale esegesi da parte dello scriba del manoscritto londinese poggiano sul fatto che, a differenza di Calfurnio, e degli altri pochi manoscritti che leggono sedebant (tutti successivi all'edizione vicentina), il codice continua a leggere mecilia facto come $\mathrm{O} \mathrm{R}$, recando un testo metricamente guasto, a fronte di un v. 4, che, recando il tuttora accolto singula, sana il singulum, lezione contra metrum di O G R. Tuttavia si noti che il testo di Catull. 113 di Add. 10386 è lo stesso della prima Aldina ed., eccezion fatta per il titolo, anche della seconda e dell'edizione di A. Guarini: quindi, a meno che sia da riconsiderare la datazione del manoscritto si possono ipotizzare due possibili approcci esegetici. 1) Il neutro plurale in quella sede non faceva difficoltà, come il commento di Guarini sembra implicitamente confermare (cf. A. Guarini Ferrariensis in C. V. Catullum Veronensem per Baptistam patrem emendatum expositiones cum indice, Venetiis 1521, f. cxiiii r., ove, a fronte di moechilia a testo, nel commento si legge

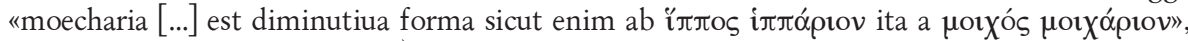
senza alcun cenno alla prosodia), sebbene Avanzi, nella Trincavelliana, torni a moechilia at di Calfurnio. 2) In realtà mal si comprendeva e pertanto si rinunciava a intervenire, come espressamente dichiara Marc Antoine Muret (Catullus et in eum commentarius M. A. Mureti, Venetiis 1554, f. 133 r: «Consule Pompeio] Ne hic quidam me pudebit fateri, hos quatuor uersus a me non intelligi. Possem, quae ab aliis adferuntur, refellere: possem ipse quoque, si liberet, aliquid eorum exemplo confingere. Malo simpliciter id quod est, dicere: neque facere, quod isti, qui dum integra, corrupta, facilia, difficilia, nusquam haesitantes, sine ullo discrimine interpretantur, imperitis fortasse docti, doctis quidem et intelligentibus imperiti atque audaces uidentur»).

${ }^{26}$ Catulli carmina cum commentario A. Parthenii, Brixiae 1486, ad loc. (sebbene la prima edizione risalga al 1485, citiamo generalmente secondo l'edizione del 1486 - M.CCCC. Lxxxvi. xi. Kal.Maias, che nei passi citati non diverge dalla prima tiratura). $35 \mathrm{r}$.

${ }^{27}$ Catullus una cum commentariis Eruditi Viri Palladi Fusci Patauini, Venetiis 1496,

${ }^{28}$ «Imilia. Ioannes Phosophorus uir tam graece quam latine bene litteratus: qui in Cypro 
Tale emendamento si può spiegare in due modi: 1) l'erudito, inserendo chilia, avrebbe inteso creare un gioco di parole, all'interno di una sorta di paretimologia, con moechilia. I "grecismi" reagirebbero tra loro, spiegando meglio la sententia finale: fecundum semen adulterio, vale a dire i moechilia hanno in sé il "germe" del chilia. Naturalmente questo è un mero tentativo di spiegare la lettura di Fosforo-Palladio: certamente con chilia si giustappone un "grecismo" a un altro che credevano di riscontrare nel testo di Catull. 2) Si è al cospetto del caso pasqualiano in cui un'edizione a stampa ci testimonia una lezione che non può essere ricondotta alla tradizione in nostro possesso ${ }^{29}$. Tale ipotesi, che, proprio per statuto pasqualiano, è indimostrabile, viene presa in considerazione per il ricorso a lezioni manoscritte ${ }^{30}$ e per i rapporti che Palladio aveva con l'Istria e la Dalmazia ${ }^{31}$ e Fosforo, "professore" a Cipro, aveva col mondo greco. In realtà ci sarebbe una terza via ${ }^{32}$ : Fosforo, venuto a conoscenza di me cilia, attraverso la lettura di manoscritti, o più verisimilmente dell'edizione di Parma o di Reggio Emilia, sarebbe stato influenzato da cilia al v. 2, che avrebbe suggerito all'erudito al v. 3 la parola greca $\chi^{i} \lambda_{\imath} \alpha$, mercé la sua somiglianza e non perché si potesse intendere al $\mathrm{v}$. 2 cilia $=\chi^{i} \lambda \lambda_{1} \alpha^{33}$.

ubi publice conductus profitebatur: nuperrime periit: legebat chilia» (Palladius, Catullus, 35 r).

${ }_{29}$ Punti 3 e 4 del "decalogo pasqualiano": 3 «è sempre probabile «a priori» che testimoni tardi dipendano, totalmente o parzialmente, da fonti diverse da quella da cui sono discesi i testimoni più antichi. Un recentior non è per ciò solo un deterior. L'autorità di un testimonio è indipendente dalla sua antichità» e 4 "quel che si dice qui di manoscritti recenti vale nello stesso modo di collazioni umanistiche e di edizioni a stampa per le quali siano stati anche soltanto consultati codici ora perduti; tranne che specialmente quest'ultimo genere di testimonianze esige particolare cautele metodiche e ingegno critico, qual è concesso soltanto a pochissimi tra i filologi» (G. Pasquali, Storia della tradizione e critica del testo, Firenze $1952^{2}$,xvi).

${ }^{30}$ Per quanto mi risulta, circa Fosforo non si hanno notizie (non escludo di tornare sulla questione in futuro). Invece Palladio è sedicente fruitore di manoscritti antichi: nota è la sua orgogliosa asserzione circa Catull. 34.3 (verso mancante in O G R): «Sed lector aduerte quod in nouis codicibus tam impressis quam manu scriptis deest tertius uersus. Quem nos in uetustiore exemplari inuentum [il corsivo è nostro] suo loco audacter reposuimus» (Palladius, Catullus, $11 \mathrm{r})$.

${ }^{31}$ Cf., e.g., J. H. Gaisser, "Catullus", Catalogus translationum et commentariorum: mediaeval and renaissance latin translations and commentaries. Volume VII, Washington 1992, 242: «Palladio Negri [...] was native of Padua, although he seems to have practiced his profession as a teacher of poetry and rhetoric chiefly in the cities of Traù (Trogir), Zara (Zadar), and Capodistria».

32 Oppure, similmente, Fosforo, leggendo una nota marginale, o interlineare, partente da me cilia, avesse introdotto al v. 3 la parola greca $\chi^{i} \lambda_{1} \alpha$. Questa "terza via", segnalatami da un referee, credo possa rientrare nella prima via illustrata sotto forma di indicazione di una delle possibili scaturigini della parola greca, purché non si creda che già cilia al v. 2 potesse essere inteso da Fosforo come mille (cf. infra n. 33).

${ }^{33}$ A meno che si voglia credere che l'umanista con duo solebant me cilia facto consule nunc iterum manserunt duo sed creuerunt milia in unum singula pensasse che due migliaia di entità imprecisate frequentassero Catullo e ora, durante il secondo consolato di Pompeo, fossero rimaste le due migliaia, che erano però cresciute di mille unità per ciascuna! 
In tutti i momenti della sua storia il testo di Catullo ha conosciuto tentativi di esegesi o di emendamento per mezzo del grecismo o di parole greche tout court. Nel XV secolo si possono individuare interventi magistrali, quali Chalybon di Poliziano in Catull. 66.48 ${ }^{34}$, oppure infelicissimi quali $x y l o p a-$ chium di Ermolao Barbaro in Catull. 53.5, ove si legge comunemente salaputium $^{35}$. Nel mezzo si registra una messe di interventi tanto suggestivi quanto difficili da dimostrare.

Forse chilia costituisce una superflua inserzione della traduzione greca in luogo del latino milia, ma proprio per questa ragione l'intervento merita una particolare attenzione: infatti se nei summentovati emendamenti il grecismo è funzionale a dipanare un testo inintellegibile, al contrario, in Catull. 113 esso si sovrappone a una parola di chiaro significato, per di più unanimemente tràdita. Fin dal XV secolo, evidentemente, il grecismo risponde a un criterio di lettura difficilior, nel caso di testo guasto, ma la lettura diventa fin troppo difficilior se il grecismo viene introdotto laddove il testo sembra già funzionare. In questo modo si tocca il limite insito anche nel criterio della lectio ${ }^{36}$

${ }^{34}$ Come l'umanista stesso riconosce, il suo emendamento non è frutto di divinazione tout court, ma il risultato di una vera e propria ricerca filologica: «Versus ex Callimacho super crinibus reginae Berenices: atque inde mendum ex Catulli exemptum uersiculo [...] Praetereaque in commentariis super Apollonium Rhodium ex Lucilio Tarrhęo Sophocleo ac Theone collectis

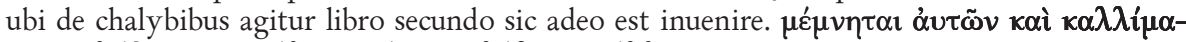

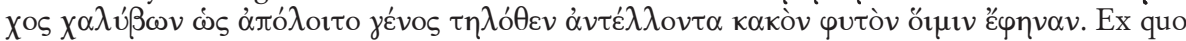
uersiculum sic illum legendum quis dubitet? Iuppiter ut Chalybon omne genus pereat: Vt sit Chalybon gręce dictum. Atque hoc inscite legunt quidam Telorum: Vulgatissimi codices celitum retinent contra etiam metri rationem. Sed hoc facio nostram obseruationem iam pridem esse praeuulgatam: quam tamen a nobis ortam uel ille ipse scit qui uulgauit libenterque etiam fatetur uir doctissimus undecumque Picus» (A. Politianus, Miscellaneorum centuria prima, Florentiae 1489, caput lxviii).

${ }^{35}$ Rimandiamo all'intera discussione dell'umanista (Castigationes Plinij Hermolai Barbari, [Venezia 1493-1494, Hain *2420], f. 62 v, ad Plin. nat. 7.29), poiché, oltre al proprio, menziona altri grecismi (solopugium, solopachium, holopachium) volti a sanare la difficile parola (salapantium V): «Huius [scil. Solipigis] diminutiuum Solopygion graece dici posse credunt: ut illud Catulli ad hunc modum legant: Dii boni solopugium disertum notata eodem uerbo et paruitate corporis et mordacitate calui Othonis sed et Solopachium quasi Holopachium defenditur a breuitate corporis cubitum totum non excedentis: Nam et Cupidines Nilotici ut est apud Philostratum Pachia uocantur. Et Lycophron Heptapachyn Achillem a spetem cubitis appellat. Ego et Xylopachium uideo legi posse: quia Xylon et Pachys mensurae sunt nomina Horus testis. Huius autem uerbi quo Catullus utitur: solus meminit: quod usque inuenerim: Seneca».

${ }^{36}$ Questo può valere, sebbene a un livello diverso, anche per gli emendamenti. Dal momento che per un referee «it is hard to see what the criterium [scil. lectio difficilior] can mean in such a context" dove "we have one transmitted reading, and another that could either be a transmitted reading or a conjecture», preciso che qui si intendeva dire che gli umanisti, al pari dei filologi moderni quando si rifanno alla lectio difficilior, dànno più chances a quanto appare meno scontato e più difficile, e che, pertanto, più le congetture sono erudite, maggiore è la loro plausibilità e il rischio che alterino il testo genuino. Similmente, nel caso di lectio difficilior può essere talvolta arduo distinguere una lezione "difficile" da una raffinata congettura. 
difficilior in termini di utilità e di plausibilità: infatti essa non deve essere superflua né tampoco improbabile.

Per poter considerare chilia "emendazione difficilior", e non gratuito sfoggio di erudizione, bisogna misurarne il "tasso di inutilità", ossia vedere se il testo con milia funziona oppure se con chilia si ha un miglioramento, oggettivo il più possibile, della lettura.

Ai fini della ricostruzione di Catull. 113 sembra che il testo e l'esegesi proposti da Palladio possano venire in aiuto, non tanto perché colgono nel segno, quanto perché sembrano introdurre un calembour, forse volontario, basato sulla lingua greca tra la prima parola del v. 2 e chilia. Infatti il neutro plurale moechilia non risolve nulla, in quanto rende necessario un intervento per correggere la sequenza di peone primo, che proprio col neutro si avrebbe in quella posizione. Inoltre, e soprattutto, una parola significante moechae paruae non rende il testo più comprensibile, anche se si opta per sedebant, in luogo di solebant, accolto, non a caso, dalle edizioni a stampa a partire dalla Vicentina per tentare di conferire un senso soddisfacente ${ }^{37}$.

Quindi non pensiamo che lo snodo di un carme che si conclude col fulmen gnomico fecundum semen adulterio sia la denuncia dell'incremento delle adultere in Roma, ma, piuttosto, l'attacco a una persona precisa, come legge G.

Invece pensiamo che sia degna di considerazione l'ipotesi di un calembour imperniato sulla lingua greca. Il gioco di parole connesso con un attacco personale è, com'è noto, pratica inveterata. Basti pensare alla sua presenza nella Commedia Antica: i nomi propri greci si prestano a giochi di parole, comici e non, per il fatto che possono essere ridotti facilmente a nomi parlanti, poiché tutti «etymologically significant $»^{38}$. Quindi non solo il potenziale etimologico del nome o di parte di esso poteva essere funzionale a un lusus, ma proprio la consapevolezza (par)etimologica della morfologia dei nomi propri permetteva di inventare nuovi nomi parlanti sul modello di nomi esistenti.

In taluni casi più che di neoformazioni si tratta di vere e proprie deformazioni finalizzate a una risemantizzazione del nome proprio di partenza. Na-

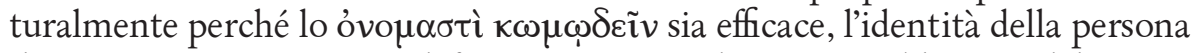
il cui nome è sottoposto a deformazione non deve essere obliterata dal lusus. Anzi, seppur a livello allusivo, essa deve essere ben presente e ricavabile dal contesto del lusus stesso. Per esempio, nell'accusa del processo ai cani di Ar.

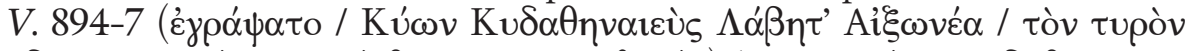

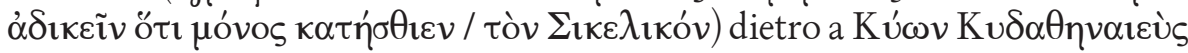

\footnotetext{
${ }^{37}$ Circa la possibilità e la volontà di dare un'esegesi al testo da parte degli editori di fine XV secolo e inizio XVI, cf. supra n. 25.

${ }^{38}$ Nikoletta Kanavou, Aristophanes' Comedy of Names. A Study of Speaking Names in Aristophanes, Berlin-New York 2011, 2. La studiosa (Aristophanes' Comedy. 2 ss.) mostra come per i nomi propri greci fosse piuttosto facile individuare l'etimologia o proporre una paretimologia.
} 
si cela uno dei favoriti bersagli di Aristofane, $\mathrm{K} \lambda \varepsilon$ ś $\omega \nu$, per l'appunto origina-

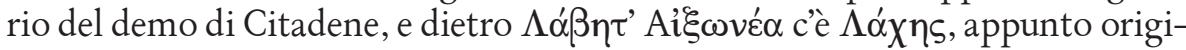
nario del demo di Aissone. Si noti come i nomi originari siano stati deformati non in neoformazioni, ma in nomi attestati ${ }^{39}$ fonicamente simili, e come rimangano decrittabili grazie all'aggettivo indicante il demo di provenienza di Cleone e Lachete. Naturalmente, una volta compresa la sostituzione di alcuni fonemi, l'aition della deformazione rende parlanti i nomi.

Si possono fare anche esempi in cui la deformazione porta a neoformazioni, ovviamente parlanti, in chiave comica: cosi Cleonimo in Ar. V.592 diven-

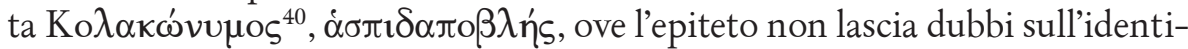
ficazione del personaggio né tampoco sullo $\sigma \kappa \tilde{\omega} \mu \mu \alpha$.

Ancora, citiamo, seppur da un contesto di matrice diversa perché non all'interno di un attacco personale, il noto facietque extemplo Crucisalum me ex Crisalo di Plaut. Bacch. 362 Questa: il passo mostra a giorno la trasfigurazione del nome originario greco X $\rho \bar{v} \sigma \alpha ́ \lambda$ os nell'assonante ${ }^{41}$ e ominoso Crŭcisalus, parola tutta latina e, ovviamente, inventata per l'occasione ${ }^{42}$.

${ }^{39}$ Per l'esistenza del raro $\Lambda \alpha \dot{\beta} \eta$ s, cf. $L G P N$ II A.

40 Tale neoformazione combina un suffisso produttivo per nomi propri con la radice di un aggettivo mai utilizzata nella composizione di nomi di persona (cf. Kanavou, Aristophanes' Comedy of Names, 95).

${ }^{41}$ L'assonanza potrebbe essere maggiore se si ipotizza che -ci- di Crucisalum potesse avere una pronuncia leggermente assibilata (sull'ipotesi è tornato recentemente M. Fontaine, Funny Words in Plautine Comedy, Oxford 2010, p. 41 n.7).

${ }^{42} \mathrm{Dal}$ momento secondo un referee che i paralleli comici non sarebbero cogenti poiché «the parallels in Aristophanes and Plautus, who had been writing centuries earlier in a different genre, do not indicate that Catullus was prone to pun on names» sono costretto a precisare che 1) Aristofane e Plauto sono evocati qui come auctores dell'attacco óvo $\mu \alpha \sigma \tau i$, noti a pubblico dotto e non di metà I a.C. (cf. per Aristofane Hor. sat.1.4.1-8 Eupolis atque Cratinus Aristophanesque poetae / atque alii, quorum comoedia prisca uirorum est, / siquis erat dignus describi, quod malus ac fur, / quod moechus foret aut sicarius aut alioqui / famosus, multa cum libertate notabant. / hinc omnis pendet Lucilius, hosce secutus, / mutatis tantum pedibus numerisque, facetus, / emunctae naris, durus conponere uersus e per Plauto cf., e.g., $O C D$ s.u. Plautus, p. 1195 «Plautus' plays continued to be performed with success at Rome at least until the time of Horace»); 2) com'è noto, gli stilemi e lessico della commedia antica e della palliata sono presenti all'interno del liber (cf., e.g., Ellis, A Commentary, passim per le citazioni e i rapporti tra poesia d'amore catulliana e palliata rintracciati già da F. Leo, Der Monolog im Drama, Berlin 1908, 109-10, confermati da studi su singoli passi e ribaditi in studi più generali -la bibliografia è davvero sterminata in modo da poterne dare conto); inoltre ipotizzare che la differenza di genere possa escludere a priori un'intertestualità in Catullo equivale a disconoscere la Kreuzung der Gattungen e degli stili propria della lingua del liber, nonché l'essenza dell'arte catulliana; 3) per la cauillatio nominis in Catullo ho dato notizia di possibili ipogrammi (cf. infra), tralasciando gli ovvi giochi di parole generati dai nomi propri Lesbia e Mentula, (diffusi, se si pensa Leuconoe e Lalage per Orazio e Delia per Tibullo) e quelli (para)etimologici coinvolgenti la lingua greca (cf. e.g. Catull. 34.15-6 notho es / dicta lumine Luna e 36.12 Vriosque apertos). 
Infine sembra opportuno fare un cenno agli studi saussuriani circa gli ipogrammi nelle letteratura greca e latina ${ }^{43}$. Infatti Saussure indagò come sovente il significante, solitamente un nome proprio, che gli autori antichi intendevano porre in evidenza, si riverberasse nei significanti vicini tramite ipogrammi (col ricorso ad allitterazioni e a sorta di anagrammi). Poco prima di licenziare questo lavoro, ho avuto modo di compulsare il recentissimo lavoro di Enrico Flores ${ }^{44}$, il quale trova in Catullo un riscontro delle teorie saussuriane: gli esempi addotti partono per lo più da nomi propri la cui trama fonica si rispecchia quasi per anagramma, nei significanti vicini ${ }^{45}$. A questi passi, vengono aggiunti altri in cui il nome proprio evocato si prolunga, non tanto mercé «automatismi psichici», quanto piuttosto consciamente, nei suoi fonemi all'interno del testo, tramite anagrammi, assonanze, allitterazioni, in modo da unire sotto un unico denominatore fonico (quello del nome proprio) altri significanti: degno di particolare nota è il caso del nome proprio Mentula in Catull. $105^{46}$.

A questo punto ritorniamo laddove ci si era interotti, ossia al problema dell'identità del personaggio di Catull. 113.2 (Mucia o Mecilia) e della grafia, nel caso si propenda per Mucia e si stampi Maecilia (o Moecilia), ma con una diversa prospettiva, quella del gioco di parole, che peraltro potrebbe risolvere una delle questioni aperte del carme, ossia l'Anrede al poeta Elvio Cinna. Infatti un poeta dotto può essere il destinatario di un lusus verbale, che peraltro, visto che interessa parole greche di uso comune, poteva essere decrittabile da una ampia parte di pubblico, visto che le commedie di Plauto, certo accessibili a un pubblico meno dotto di Elvio Cinna, sono ricche di nomi parlanti greci.

\footnotetext{
${ }^{43}$ Oggetto di una recente analisi da parte di D. Bruzzese, "La ricerca anagrammatica di Ferdinand de Saussure: un'altra riflessione sul segno”, RIFL 3, 2010, 65-77 (URL: http://www.rifl. unical.it/articoli/rifl032010/007Bruzzese.pdf), alla cui bibliografia ci permettiamo di rimandare (cf. pp. 67 ss.).

${ }^{44}$ E. Flores, "Il significante ermetico, Saussure e la scoperta dell'inconscio nella scrittura", Vichiana 14, 1, 2012, 3-47.

${ }^{45}$ Vengono citati svariati passi, degni di menzione ci sembrano quelli che coinvolgono i nomi propri: in Catull. 69.1-2 e 77.1-2 a partire da Rufus il poeta organizza una particolare trama fonica (in particolare nel c. 69 femur «contiene l'anagramma dell'incipitario vocativo $R u f e$ con in più una $m$ centrale che riprende il precedente rum» di tenerum e "la catena degli stessi fonemi in Rufe-rum femur è per di più evidenziata nel pentametro dai tre ictus sulla $u »$; cf. Flores, "Il significante ermetico", 6-7); l'anagramma di Aureli si può trovare nascosto nei fonemi di Catull. 21.1-3 e un'articolata tessitura fonematica a partire da Varus è riscontrata in Catull. 10.1-2.

${ }^{46}$ In Mentula conatur Pipleum scandere montem / Musae furcillis praecipitem eiciunt «il distico elegiaco è contrassegnato, oltre che dall'anagramma di Mentula contenuto nel primo verso, da un ricchezza di difoni fonematici $(t u)$, allitterazioni (Men- mon- Mu-) anche all'interno dei due versi, assonanze (-um -em 2 volte almeno nella scrittura)» (Flores, "Il significante ermetico", 13).
} 
Quindi esaminiamo nuovamente le due identità:

1) Se il personaggio è la per noi sconosciuta Mecilia non è immediato pensare a un qualche gioco di parole costruito con milia, chilia o qualche altro significante di Catull. 113. Infatti il greco chilia (oppure $\left.\chi^{i} \lambda_{\imath} \mathbf{\alpha}\right)$ mal si combina con Maecilia, poiché costringe a pensare retroattivamente a una pronuncia grecizzante Maechilia di un nome latino. In questo modo, forse, il calembour giocato col duo e chilia funzionerebbe: due frequentano una Mecilia, dopo quindici anni sono rimasti due, ma sono cresciuti per chilia, perché una Mae-chilia funziona da moltiplicatore per mille, visto che fecundum semen adulterio. Così il personaggio ignoto risulterebbe esser stato scelto per il potenziale ominoso del suo nome, che però funziona solo se lo si legge "alla greca". Ma, that is the question, perché siffatta pronuncia? Forse che Mecilia è moglie di un personaggio che, similmente ad Arrio ${ }^{47}$, inseriva occlusive aspirate, in questo caso in posizione centrale, per avere una pronuncia elegantemente grecizzante ${ }^{48}$ ? Oppure lei stessa aveva tale vezzo? Solo con questa, o una qualsivoglia altra, lucubrazione, la parola avrebbe ragion d'esser "greca" e potrebbe diventare in una delle sue parti "parlante". A ben vedere quindi il gioco di parole non risolve, anzi amplifica, i problemi connessi con Mecilia e, inoltre, risulterebbe un poco legnoso se non forzato.

2) Se il personaggio è Mucia, la cauillatio nominis può spiegare l'alterazione del nome che deve essere supposta qualora si accetti il dato codicologico. Qualora invece non lo si accetti, si ricorre a Mucilla, nome di formazione regolare, ma hapax, che, non si presta a nessun lusus, né con milia, né con chilia o con un altro significante di Catull. 113, sicché il tentativo di restituire un diminutivo regolare di Mucia sembra escludere una cauillatio nominis.

Se quindi si ipotizza un calembour, il tràdito mecilia $(m)$ è forse molto meno corrotto di quanto pensano alcuni sostenitori della presenza di Mucia.

${ }^{47}$ Per quanto ne sappiamo Arrio tendeva a porre aspirazione all'inizio di parola e non altrove, quindi si potrebbe escludere un coinvolgimento di Arrio in questo testo. Tuttavia se Arrio è veramente Quinto Arrio, citato in Cic. Brut. 242 (generalmente si dà per più probabile questa identificazione rispetto ad altre), si potrebbe trovare nella menzione dei due consolati di Pompeo un appiglio per la rocambolesca spiegazione di Maechilia quale moglie di Arrio, dal momento che Cicerone afferma che fuit M. Crassi quasi secundarum (Brut. 242) e Crasso era il collega di Pompeo in entrambi i consolati. Tuttavia, come si vede, più si avanza e più gli appigli in questa direzione sono scivolosi e fuorvianti.

${ }_{48}$ Non si intende prendere posizione circa quale origine avesse la pronuncia di Arrio (rustica, nel caso di identificazione con Quinto Arrio, o grecizzante), la quale in ogni caso era dallo stesso ricercata per avere una "meravigliosa" dizione ('Chommoda' dicebat, siquando commoda uellet / dicere, et insidias Arrius 'hinsidias', / et tum mirifice sperabat se esse locutum, Catull. 84.1-3). Dal momento che si sta valutando la possibilità di calembour basato sul greco, è ovvio che il bersaglio doveva aspirare (è proprio il caso di dirlo!) a una pronuncia greca perfetta e quindi estendeva per analogia l'aspirazione anche a occlusive sorde. Com'è noto, tale estensione analogica a partire dal greco è responsabile del cambiamento della pronuncia di alcune occlusive sorde in corpo di parole latine o imprestiti dal greco (cf., e.g., J. N. Adams, Bilingualism and the Latin language, Cambridge 2003, 109 e bibliografia ivi citata). 
Infatti Pleitner ${ }^{49}$, che per primo suggerì di identificare Mucia nel nome proprio di Catull. 113.2, suggeriva di emendare in Mucillam, non potendo evidentemente spiegare una sua alterazione. Invece alcuni suoi epigoni, intendendo giustificare la lezione della stragrande maggioranza dei codici, hanno fornito motivazioni linguistiche che possono essere riconsiderate alla luce dell'ipotesi del lusus verbale.

Friedrich ${ }^{50}$ ha tentato di giustificare mecilia di O R m quale corruttela di Mucilla: il finale -ilia per -illa sarebbe imputabile sia alla facilità dello scambio -li- / -ll- (attestato anche nei manoscritti catulliani), che adombrerebbe un'effettiva pronuncia $l i$ per la doppia $l$, sia al tenore difficilior dello hapax Mucilla rispetto all'attestato $M(a)$ ecilia, il dittongo oe in luogo di $u$ sarebbe una variante ortografica ${ }^{51} \mathrm{e}$ la forma ablativale andrebbe preservata sulla scorta di un passo terenziano, sottointendendo uti.

Crediamo che la giustificazione del dittongo in luogo di $u$ e del finale -ilia al posto di -illa quali solecismi non sia del tutto soddisfacente. Infatti, l'ipotetica selezione di una determinata variante diastratica, se da una parte può alludere al fatto che il nome e le gesta di Mucia fossero sulla bocca di tutti (amanti e non), dall'altra non trova piena giustificazione, specie in un carme indirizzato a Cinna, il cui bersaglio è Pompeo. Invece una pronuncia solecistica del nomignolo, specie se "vulgata", può aver concorso alla creazione di un qualche lusus linguistico.

Rientra così in gioco l'esegesi degli umanisti che connettevano moechilia (o simili) ${ }^{52}$ con $\mu$ orxó $\varsigma^{53}$ e corradicalii ${ }^{54}$. Naturalmente non crediamo che la

${ }^{49}$ K. Pleitner, Des Q. Valerius Catullus Epigramme an und über C. Jul. Caesar und Mamurra, Speyer 1849, 22-3. Che il nome del v. 2 adombrasse un'amante di Pompeo era già circolante ai tempi di Alessandro Guarini: «aliqui hoc conentur referre ad floram scortum quod amauit Pompeius et geminius eius familiaris, cum postea eadem multos habuerit amatores, ut in eius uita legitur [Plu. Pomp. 2,2-8]" (Guarini Ferrariensis in C.V. Catullum Veronensem per Baptistam patrem emendatum expositiones, f. cxiiii r.).

${ }^{50}$ Catulli Veronensis liber, erklärt von G. Friedrich, Leipzig-Berlin 1908, 547-8

${ }^{51}$ Già Baehrens, Catulli Veronensis liber, recensuit et interpretatus est Aem. B., volumen alterum, Leipzig 1885, 603, ritiene Moecilla una «forma plebeia» rispetto a Mucilla.

${ }^{52}$ Si ricordino anche moechalia di Partenio e moecharia di A. Guarini (cf. supra n. 25).

${ }^{53}$ Più recentemente anche M. Ruiz Sánchez, Confectum carmine. En torno a la poesía de Catulo, Murcia, 1996, II, 356 riprende la connessione di Maechilia con $\mu$ orxท́: «el artificio del poema se basa en la presencia de una segunda isotopía y no sólo en la antítesis. Esta segunda isotopía es de carácter económico. La idea que sirve de base al texto es la del «rendimiento» del adulterio. Se hace "balance» de los amantes de la mujer atacada [...] El «interés» que el tiempo ha aportado ha sido elevado (mil por uno) y el texto concluye: fecundum semen adulterio. Esta es la "punta" del epigrama, donde confluyen las dos isotopías. La imagen agrícola no nos aparta de la idea principal, sino al contrario [...]. El matrimonio de Mecila no ha producido hijos, sino adulterios».

${ }^{54}$ Tale esegesi peraltro, venne ripresa, dopo la dichiarazione di rinuncia di Muret (cf. supra n. 25), da Achille Stazio (Catullus cum commentario Achillis Statii Lusitani, Venetiis 1566) che al v. 2 emendava moechi illo facto etc. e dallo Scaligero (Catulli, Tibulli, Properti, nova editio) moechi illi, ah, facto etc.; fino alla prima edizione di Lachmann ( $Q$. Valerii Catulli 
parola sia un neutro plurale. Tuttavia l'afflato adulterino di Mucia può giustificare la sua alterazione in un improbabile grecismo modellato su moech a che un certo tipo di pronuncia poteva suggerire.

Non è improbabile che la sillaba Mu-di Mucia potesse essere distorta in Moe- di moecha, a causa di una sorta di contiguità fonica nel latino, sia in particolari varietà diastratiche, sia a livello di Langue a causa delle alternanze presentate da parole corradicali. Infatti per alcune radici sono attestate vocalizzazioni alternanti oe/ $u$ : si pensi a moenia a fianco di parole corradicali come munire, ma anche a poena confrontata con punire e a Poeni con Punicus. Ancora, il dittongo oe nel latino classico figura, com'è noto, solo nei prestiti greci contenenti il dittongo greco or e in nove parole latine $e^{55}$, mentre a fronte di un latino arcaico oi si ha quasi sempre la chiusura del dittongo in una $u$ lunga. Se, alla luce di moecha, sembra giustificabile la sillaba $m e-$ della tradizione antiquiore all'interno della prima parola del v. 2, resta da esaminare il -cilia tràdito. All'uopo potrebbe essere davvero decisivo l'uso di $x^{\prime} \lambda_{1} \mathbf{} \alpha$ in luogo di milia: infatti la sola somiglianza tra due parole non può bastare a rendere limpido e immediato un calembour basato su una neoformazione, che ha bisogno, per suo statuto, di essere resa decrittabile da qualche altro elemento, in questo caso l'apparentemente gratuito chilia di Fosforo in luogo di milia. La parola greca chilia/ $\chi^{i} \lambda_{\mathbf{\imath}} \alpha$ potrebbe fornire la chiave di lettura per lo strano nome al v. 2 , poiché attraverso una sorta di adnominatio si collega a esso fornendone l'origine e il significato. Si pensi infatti, ad esempio, all'adnominatio di Plaut. Bacch. 240 Questa opus est criso Crisalo: è chiaro che la scelta della parola greca si giustifica col solo gioco di parole, attivando il potenziale semantico dell'etimo del nome di persona che sembra rendere assurda l'asserzione, peraltro pronunciata da Crisalo stesso.

Quindi se si prova a vedere in meciliam di G un non improbabile, almeno dal punto di vista paleografico, Moechiliam, chilia / Xí $\lambda_{\mathbf{1} \alpha}$, in luogo dell'atteso milia, suggerisce una sovrapposizione-sostituzione di parole latine con parole greche fonicamente vicine. Così Moechilia dal

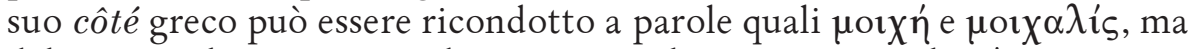
dal suo côté latino a nomi di persona quali Mucia o, meglio, ${ }^{*}$ Mucilla. A supportare queste "due anime" di Moechilia rientra in gioco il contesto del carme, con i suoi riferimenti ai consolati di Pompeo e con la sua sententia finale concernente l'adulterio. Infatti tali informazioni inducono a pensare alla moglie fedifraga di Pompeo, che risponde al nome di Mucia (e che moecha, almeno per Pompeo, doveva essere), tanto facilmente che lo si è già fatto indipendentemente dall'interpretazione del nome proprio del v. 2.

Veronensis liber, Berlin 1829) tali soluzioni saranno le più accettate.

${ }^{55}$ Cf., e.g., M. Leumann, Lateinische Laut- und Formenlehre, München, 1977, 65-7. 
Si noti che in questo modo la tensione tra il latino Mucia e l'improbabile greco Moechilia crea un ulteriore gioco di parole: infatti Moechilia, più che a Mucia, sembra rimandare a un non attesato Mucilla, che si può giustificare quale diminutivo. Se in passato trovava argomentazioni circa la propria inopportunità, ora il diminutivo si giustifica in chiave ironica, visto che il lusus lo trasformerebbe in una sorta di composto greco di $\chi^{i} \lambda^{\prime} \mathbf{t o v}$ : una Mucia, matrona ipocoristicamente denotata dal suffisso di diminutivo, risulta trasfigurata in una Morx $\left(\eta^{\prime}\right)-\chi i \lambda_{1} \alpha$ cioè in un donna mille volte moecha.

L'ipotesi può sembrare azzardata, tanto più che una tale esegesi greca di Moechilia prescinderebbe dalla prassi secondo cui il tema di $\chi^{\mathbf{i}} \lambda \mathbf{i o r}$ è la prima parte della parola. Cionondimeno mecilia $(m)$ della tradizione sembra funzionare pienamente quale allusione a Mucia, solo se lo si intende come "parola greca". Proviamo dunque a leggere ipoteticamente Catull. 113 col seguente testo, ossia accogliendo al v. 2 la deformazione Moechilia e l'emendamento di Fosforo al v. 3:

\section{Consule Pompeio primum duo, Cinna, solebant Moechiliam: facto consule nunc iterum manserunt duo, sed creuerunt $\chi^{i} \lambda_{1} \alpha$ in unum singula: fecundum semen adulterio!}

Ossia, durante il primo consolato di Pompeo duo solebant Moechiliam e durante il suo secondo consolato manserunt duo sed creuerunt $\chi^{i} \lambda_{1} \alpha(\mathrm{e}$ non l'atteso latino milia) in unum singula: la presenza di $\chi^{i} \lambda_{1} \alpha$ permette non solo di rendere Moechiliam intelligibile quale succedaneo "greco" di parola latina, ma anche di avere una sorta di corrispondenza parafonica e paretimologica tra le due "parole greche". Infatti manserunt duo sed creuerunt $\chi^{i} \lambda_{1 \alpha}$ ha il suo pendant nell'antefatto duo solebant Moechiliam: i $d u o$ restano ma sono cresciuti mille volte per ciascuno. Anzi più che cresciuti, dovremmo dire moltiplicati, quasi che i $d u o$ entrando in contatto con una Moechilia si siano moltiplicati per $\chi^{i} \lambda^{\prime} \mathbf{l} \alpha$. Indubbia peraltro è la volontà di giocare coi "numeri" (a parte i due "grecismi" nell'ordine si ha: primum, duo, iterum, duo, unum, singula) e come sia forte la pressione sui significanti. In particolare $d u o$, in entrambe le occorrenze, è messo in rilievo dalle incisioni ${ }^{56}$.

Del resto la sententia finale sembra suggerire proprio che l'adulterio, quasi fosse una pianta ${ }^{57}$ ha un seme particolarmente fertile (cf. v. 4 fecundum semen adulterio $)^{58}$. Proprio la juón

${ }^{56}$ Così, e.g., J. Ferguson, Catullus, Lawrence 1985, 339.

${ }^{57}$ Cf. Kroll, C. Valerius Catullus, 285.

${ }^{58}$ Addirittura c'è chi, cogliendo la metafora del mondo vegetale, ha pensato che «the poem end with a'mock-proverb' playing on the senses of creuerunt and semen: crescere is used for growing seed (semen), but is also used to mean 'grow to manhood [...] and semen obviously in this context has the meaning of 'sperm'» (J. Godwin, Catullus, the shorter poems, Warmin- 
tutto sarebbe da intendere così: se duo frequentano Mucia cioè una Moechachilia, passato un po' di tempo, accade che gli amanti sono diventati duemila in virtù del fatto che l'adulterio ha un semen fecundum.

Quindi Moechiliam, ricavabile da G, chilia di Fosforo, il riferimento insistito a Pompeo, alla fecondità dell'adulterio, e l'ipotesi che al v. 2 il poeta alluda a Mucia, possono tenersi insieme.

Infatti, una volta chiarito il significato greco di Moechilia, mercé $\chi^{i} \lambda_{\imath} \mathbf{} \alpha$, alla fine il fruitore del carme potrà avere una conferma dei suoi sospetti: infatti chi è quella adultera il cui nome è assonante a Moechilia e che al tempo del primo consolato di Pompeo aveva due amanti? L'insistenza del carme su Pompeo e la consonanza di Moechilia con Mucilla sembrano indurre a pensare a Mucia, terza moglie del Magno, che questi aveva ripudiato a causa dei frequenti adulteri.

Resta semmai da affrontare la pars destruens di chi non crede alla presenza di Mucia in Catull. 113, rilevando incoerenza fra le date del divorzio e dei consolati di Pompeo. Durante il suo primo consolato, 70 a.C., Pompeo era già da qualche tempo sposato con Mucia, probabilmente da una decina d'anni, e sappiamo che il divorzio avvenne nel 62 a.C., motivato da adulteri commessi negli anni precedenti ${ }^{59}$. Stando a Svetonio una delle tante amanti di Cesare era proprio Mucia ${ }^{60}$ : non è improbabile ipotizzare che Catullo, anche solo per malignità, affermasse che nel 70 Mucia avesse relazioni con due persone, una, il marito, e l'altra, Cesare. Nel 55 a.C., secondo consolato di Pompeo e data del componimento del carme (cf. v. 2 facto consule nunc), i due "frequentatori" di Mucia del 70 a.C. sono cresciuti, moltiplicandosi ognuno per mille: pace Fordyce e Thomson non fà nessuna difficoltà parlare nel 55 a.C. dell'incremento degli amanti di Mucia, anche se Pompeo si era separato da costei sette anni prima. Anzi, il coinvolgimento di Cesare, verso cui gli attacchi sono uno dei Leitmotiven degli scommata catulliani, associato al gioco di parole dato da Moechilia, rende possibile un'interpretazione metaforica della moltiplicazione. Dal 70 al 55 pompeiani e cesariani sono aumentati grazie alla malversazione, di cui Mucia, alias Moechilia, può essere una delle migliori ipostasi

\footnotetext{
ster 1999, 220).

${ }^{59}$ Questa è l'ipotesi comune, mentre le fonti sembrano un poco più sibilline: infatti Plu.

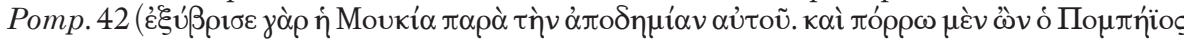

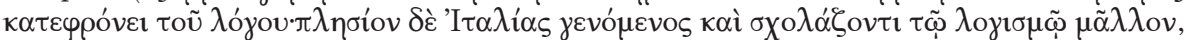

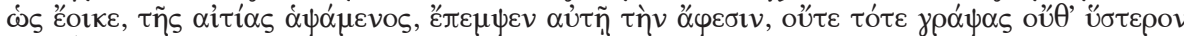

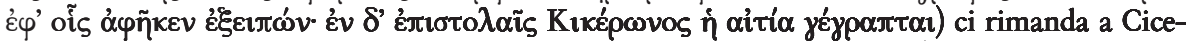
rone dal quale tuttavia non riusciamo a trovare informazioni al riguardo (cf. D. R. ShackletonBailey, Cicero's letters to Atticus, volume I (books I-II), Cambridge 1965, 299 a proposito di Cic. Att.1,12,3 Pompeium nobis amicissimum constat esse. diuortium Muciae uehementer probatur).

${ }^{60}$ Cf. Suet. Caes. 50.1 pronum et sumptuosum [scil. Caesarem] in libidines fuisse constans opinio est, plurimasque et illustres feminas corrupisse, in quibus Postumiam Serui Sulpici[i], Lolliam Auli Gabini, Tertullam Marci Crassi, etiam Cn. Pompei Muciam.
} 
insieme a Mamurra (cf. Catull. 29). Infatti, similmente a Mentula, Mucia diventa trait d'union tra Pompeo e Cesare e simbolo della loro corruzione: non si dimentichi che Pompeo, una volta ripudiata la terza moglie, si sposò per la quarta volta nel 59 a.C., con una figlia dell'amante dell'ultima moglie: cioè Giulia, figlia per l'appunto di Cesare. Mucia quindi è il pretesto per attaccare Pompeo (e Cesare) nel 55 con la pointe finale fecundum semen adulterium che in quegli anni poteva essere forse anche più ricca di implicazioni politiche rispetto al periodo del ripudio di Mucia.

Così Moechiliam, associato a chilia di Fosforo-Palladio, si potrebbe configurare come tentativo di giustificare un nome, sul quale finora non è stata fatta piena luce, perché, a seconda delle interpretazioni, mecilia $(m)$ della tradizione comporta ora l'introduzione di un personaggio femminile altrimenti ignoto, ora una grafia peregrina e altrimenti ingiustificabile per Mucia.

Certamente è vero che una tale spiegazione, imperniata su un non semplice gioco di parole, potrebbe apparire un mero intellettualismo, che in quanto tale si addice di più all'esegeta che al poeta. Tuttavia un lusus, forse proprio perché piuttosto lambiccato, potrebbe però gettare luce sulla "dedica" del carme a Cinna, ossia verosimilmente Elvio Cinna, neotero sodale di Catullo. Infatti, di per sé, Mucia non doveva destare particolare interesse nel dedicatario, almeno per quanto ne possiamo sapere. Quindi l'Anrede a Cinna, come quella a Catone del carme 56 , non si può giustificare solo come presentazione di un fatto ridicolo al quale i poeti sodali erano interessati ${ }^{61}$, ma anche e soprattutto per la presenza di un paignion che nel 56 è da vedere nella citazione archilochea, e qui potrebbe consistere invece in un elaborato gioco di parole.

Una tale lettura dunque farebbe di Catull. 113 un attacco nei confronti di Mucia e, soprattutto, nei confronti dei due "frequentatori" di Mucia: Pompeo e Cesare ${ }^{62}$. Come si è detto, Mucia sarebbe lo snodo e il tramite, metaforico (ma non solo), delle malversazioni dei due, un po' come Mamurra nel carme 29 è lo strumento con cui Cesare e Pompeo depredano Roma (cf. Catull. 29.11-24). Orbene, c’è chi, indipendentemente da Fosforo-Palladio e dalla so-

${ }^{61}$ Come invece ipotizzava Kroll, C. Valerius Catullus, 285. Altri invece giustificano la dedica a Cinna con una componente metapoetica: H. P. Syndikus, Catull, eine Interpretation. Dritter Teil. Die Epigramme (69-116), Darmstadt 1997, 136 la individua, nei carmi 56 e 113, proprio nella loro ostentata assenza di contenuti "impegnati”, la Skinner nella consapevolezza del poeta dell'applicazione di un determinato espediente (Catullus in Verona, 137: «Metonymic linkage between various forms of sexual irregularity and tainted, opportunist politics has served as the controlling trope of the elegiac epigrams. We come now to poem 113, which adopts an ironic, distanced stance toward that trope. The fact that Catullus addresses Cinna, his literary sodalis, indicates that this is a self-conscious poetic stratagem").

${ }^{62}$ Naturalmente il potenziale politico del carme è già stato rilevato (cf. e.g. C. Deroux, "L'attitude politique de Catulle", Latomus 29, 1970, 615). Si noti che L. Schwabe, G. Valerii Catulli liber. Voluminis prioris pars prior quaestionum catullianarum liber I, Gissae 1862, 220, ritiene che i due amanti in questione siano Cesare e Mamurra. 
miglianza di meciliam di $\mathrm{G}$ con moecha, ha creduto di poter riconoscere, altrove, cioè in Catull. 94, un lusus verbale sempre imperniato su Mucia e la radice di $\mu$ oıxós, e, per di più, connesso con Mentula / Mamurra.

Si prenda il testo di Catull. 94, come è stampato dal più recente editore di Catullo:

\section{Mentula moechatur, moechatur mentula certe; hoc est quod dicunt: 'ipsa olera olla legit'.}

Gli editori moderni interpretano il chiasmo mentula moechatur moechatur mentula, come giustapposizione di due proposizioni, o entrambe affermative o la seconda interrogativa alla quale alcuni rispondono col solo certe, altri con certe hoc est quod dicunt etc. ${ }^{63}$. Il testo presenterebbe una situazione, che, apparentemente particolare, si rivela proverbiale (cf. v. 2 hoc est quod dicunt: ipsa olera olla legit). Ciò che finora ha attratto per lo più la critica è l'origine e il preciso significato del detto, poiché, a quanto pare, sarebbe attestato solo da Catullo.

La comparsa di Mucia nel carme è stata ipotizzata da Baehrens, che suggeriva, nel suo commento, di leggere Moechula in luogo del secondo mentula di Catull. 94.1, perché poco soddisfatto dell'esegesi comune: «hinc cum $\mathrm{O}$ praebeat 'metula', suspicatus sum latere 'mecula' siue 'Moechula', intellegens Pompei uxorem Muciam» ${ }^{64}$.

Infatti tra le varie spiegazioni si è imposta quella tuttora accettata, secondo cui il doppio valore di mentula sarebbe alla base dell'espressione proverbiale citata. Quindi se a moechari è un uomo che si chiama mentula, la situazione sarebbe quella dell'olla che raccoglie da sé gli olera. Tale esegesi sembra derivare dall'intuizione di Achille Stazio, il quale credeva che «Sententia uero eiusmodi est, ut, cum Mentulam hunc, siue quis alius, adulter esset insignis, cum Catullus aut se ipse sic exscusare fingatur, se non moechari sed mentulam

${ }^{63}$ 1) due affermazioni: W. Eisenhut, Catulli Veronensis Liber, Leipzig 1983: 'Mentula moechatur.' moechatur mentula certe. / hoc est, quod dicunt etc. (cf. H. Bardon, Catulli Veronensis Carmina, Stuttgart 1973²: Mentula moechatur. moechatur Mentula certe. / hoc est quod dicunt etc. e E. D'Arbela (a cura di), Catullo. I carmi, edizione critica e traduzione, Milano 1957: Mentula moechatur; moechatur mentula certe. / hoc est quod dicunt etc.). 2) un'affermazione seguita da una domanda, con due diverse punteggiature della relativa risposta: a) Della Corte, Catullo: Mentula moechatur. "Moechatur mentula?» Certe / hoc est quod dicunt etc. (cf. Thomson, Catullus: Mentula moechatur. moechatur mentula? certe / hoc est quod dicunt etc. ) e b) G. P. Goold, Catullus: Mentula moechatur. 'moechatur mentula?' certe. I hoc est quod dicunt etc. (cf. R. A. B. Mynors, C. Valerii Catulli carmina, Oxford $1958=\mathrm{La}-$ faye, Catulle: Mentula moechatur. Moechatur mentula? Certe. / hoc est quod dicunt etc.).

${ }^{64}$ E. Baehrens, Catulli Veronensis liber, volumen alterum, 575-6. Tale emendamento era già stato anticipato in apparato nella sua edizione del 1876: «malim Moecia (Mucia) uel Moechula", laddove accoglieva a testo la vulgata ripetizione di mentula (Catulli Veronensis liber, recensuit et interpretatus est Aem. Baehrens, volumen prius, Leipzig 1876, 104) 
ipsam, in suamque mentulam sic culpam conferat, ut uulgo dici etiam solet, non olera aliquem furatum, sed ollam ipsam» ${ }^{65}$.

Tuttavia i dubbi di Baehrens circa la ricostruzione del detto proverbiale ${ }^{66}$ e il suo giudizio del calembour Mentula-penis ${ }^{67}$ sembrano motivati: infatti, con tale esegesi, il carme si riduce a un'affermazione Mentula moechatur che viene giustificata, ripetendo il significante mentula, attraverso un chiasmo ottenuto con le medesime parole e con la collocazione in posizione di rema del sostantivo. Se è vero che il carme 94 si basa implicitamente sul soprannome di Mamurra, non è improbabile che il carme sia posteriore ad altre invettive catulliane, in particolare a quella di Catull. 29, ove si ritiene che il soprannome Mentula sia stato elaborato e motivato. Se è così, non si capisce quale sia la particolarità di Mentula moechatur, a meno che si voglia credere che Catullo abbia scritto il distico per ricordare che Mamurra è una mentula fatta persona e per questo il suo moechari è giustificato in quanto, se si si dà credito ad Achille Stazio, Mamurra, potendo essere assimilato e ridotto essenzialmente alla "parte" che moechatur, finisce per essere colpevole, ma, allo stesso tempo, non condannabile, come l'olla del v. 2. Anche qualora l'asserzione mentula moechatur venisse proposta sottoforma di domanda, nulla sembra suggerire cosa, appunto, ci sia di strano in tale frase.

Quindi la frigiditas, rilevata da Baehrens, non sembra consistere in qualcosa di soggettivo, ma forse soprattutto, nel fatto che il poeta si limita a ribadire, quasi tautologicamente, l'efficacia del soprannome Mentula. Ancora, è tutt'altro che sicura la pur ingegnosa spiegazione di Achille Stazio, proprio perché legere, per quanto possa avere il valore più specifico di "rubare", trova il suo pendant nel moechari e perché all'olla sembra corrispondere mentula. Prima di tutto esiste una tradizione letteraria e paremiografica per la situazione in cui un individuo per scusarsi accusa o una singola parte del suo corpo o uno strumento di cui si è servito ${ }^{68}$ : se l'esegesi di Achille Stazio cogliesse nel segno, bisognerebbe constatare che il poeta allude a questa tradizione senza sfruttarla e, soprattutto, vi allude con un'espressione proverbiale che sembra addirsi poco a Mentula moechatur.

Infatti detto strumento (l'olla) e l'azione che compie (legere per riempirsi) hanno poco a che spartire con una mentula: tutt'al più si possono trovare

${ }^{65}$ Catullus cum commentario, 387

${ }^{66}$ "quae omnia ut incerta sunt (uelut de furis excusatione ex olla petita), ita a uerborum conformatione uix quidquam accipiunt praesidii» (Baehrens, Catulli Veronensis liber, volumen alterum, 575).

${ }^{67}$ «abhorret animus, quod perquam frigidus est lusus 'Mamurra moechatur, moechatur eius penis', quocumque modo intellegimus, idque eo magis quod ob penem Mamurrae dedit cognomen Mentulae Catullus ipse» (Baehrens, Catulli Veronensis liber, volumen alterum, 575).

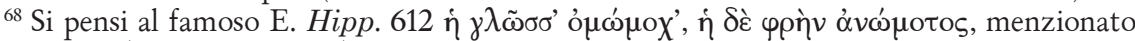
da Aristotile (Rh. 1416 a 28 ss.) e variamente parodiato e citato (cf., e.g., C. Austin - S. D. Olson, Aristophanes Thesmophoriazusae, edited with introduction and commentary, Oxford 2004, 143). 
punti di contatto molto generici, quali il moechari accostato al legere e i due "strumenti" che diventano gli autori e responsabili di azioni all'interno delle quali assolvono di solito una funzione meramente strumentale. Anzi, a ben vedere, l'azione della mentula all'interno del moechari e dell'olla nell'olera legere si differenziano, almeno in un punto, addirittura diametralmente: tanto è fisiologica la presenza di una mentula all'interno di un adulterio, quanto impossibile è un'olla che, motu proprio, raccoglie/ruba. Quindi perché giustificare un fatto assodato e banale come un Mentula e una mentula che moechatur con un'azione che, per quanto proverbiale essa sia, è paradossale e ovviamente impossibile ${ }^{69}$ ? Infatti il doppio significato di Mentula giustifica il moechari almeno su un piano, per così dire, di natura, che non è quello del proverbiale ipsa olera olla legit, che sembra poggiare su un paradosso.

A meno che si voglia credere con Paul Claes che il genere grammaticale femminile associato a moechari ${ }^{70}$ comporti che la situazione del proverbio sia perfetta poiché «this man [scil. Mamurra] designated by a feminine term must be an effeminate i.e. a passive homosexual», come proverebbe anche Catull. 57, "where Mamurra is called his [scil. Caesar's] pathicus». Pace Claes, generi grammaticali e differenze sessuali non vanno sempre di pari passo ${ }^{71}$. Mentre nel carme 57 con cinaedis / Mamurrae pathicoque Caesarique (vv. 1-2) il poeta sicuramente definisce cinaedus Mamurra, ma l'epiteto può rimanere entro i limiti dell'insulto ${ }^{72}$ e non essere un'accusa. Per quanto riguarda $p a-$ thicoque, l'aggettivo rimane in modo ambiguamente graffiante $\alpha$ đò kovvoũ tra Mamurra e Cesare ${ }^{73}$. Infine Claes presuppone che l'occorrenza catulliana

${ }^{69}$ Cf. già G. Damschen, “Catullus c. 94: «ipsa olera olla legit»”, Mnemosyne 52, 1999, 173: «wenn ein Mann dieses Namens [scil. mentula] oder der entsprechende Körperteil überhaupt Unzucht treibt: Das ist doch - mehr oder weniger — seine natürliche Bestimmung!»

${ }^{70}$ P. Claes, "Catullus c. 94: the penetrated penis", Mnemosyne 49, 1996, 66: «With a masculine subject the verb moechari means 'to have a mistress, to be a seducer'; with a feminine subject it means 'to be a mistress, to be seduced'. The feminine term mentula calls for a passive meaning of moechari: to act like a moecha, a seduced wife». Si noti che la forma deponente è caratteristica di verbi denominali della prima coniugazione col valore di «"sich betätigen", "benehmen als"”, da intendersi forse quale grecismo e, nel caso di moechari, il modello sembra essere stato proprio il verbo greco corrispondente $\mu \mathrm{ol}_{\chi} \tilde{\alpha} \sigma \theta \alpha \mathbf{l}$ (cf. Leumann, Lateinische Lautund Formenlehre, 546).

${ }^{71}$ Per taluni sostantivi il genere naturale non aiuta a studiare l'evoluzione di quello grammaticale, come, e.g., per le testimonianze circa il genere di mus (cf. S. Boldrini, "Topo/topa: una questione di genere", Paideia 59, 2004, 43-50, vd., in particolare, il problema del genere di mus in Plin. nat.10.185, cui lo studioso fa cenno a p. 44).

${ }^{72}$ Questa argomentazione è già stata addotta da Damschen, "Catullus c. 94", 172. I commentatori registrano qualche incertezza nel rubricare la parola quale epiteto ingiurioso e non quale accusa: e.g. H.P. Syndikus, Catull, eine Interpretation. Erster Teil. Die kleinen Gedichte (1-60), Darmstadt 1984, 277 n. 6 deve specificare che «Wenn der Beziechnung cinaedi in der nächste Zeile das praktisch synonyme [sic] pathicus nachgeschoben wird, bringt das keine zusätzliche Information, sondern ist eben ein weiteres Schmähwort». Invece non sembra essere esclusa la presenza dell'accusa oltre all'insulto in Thomson, Catullus, 340-1.

${ }^{73}$ Peraltro tutto il carme è imperniato sulla condivisione di ogni cosa da parte dei due pro- 
di moechari abbia un valore generico di "sedurre" 74 , mentre propriamente denota un atto adulterino (cf. ThLL 8.1322 .81 ss.). Mancando la nozione di adulterio la domanda del carme sarebbe ancor più vana: dal momento che il lusus paragrammaticale ipotizzato non convince, l'ipotesi esegetica di Claes non sembra sufficiente a conciliare mentula moechatur con olera olla legit.

Più documentato il tentativo di Gregor Damschen, secondo cui «Das Sprichwort macht deutlich, in welche Gefahr sich Mamurra mit seinem ehebrecherischen Verhalten begeben hat» ${ }^{75}$, ossia Mamurra, col suo moechari, avrebbe provocato la propria punizione. Infatti ipsa olera olla legit vorrebbe dire che le terga di Mamurra hanno scelto da sé e volontariamente la

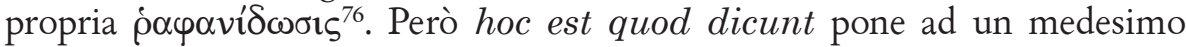
livello l'azione del v. 1 con la sententia del v. 2 : se così fosse, il poeta avrebbe voluto dire che l'adulterio corrisponde alla scelta della punizione prevista per esso. In realtà il moechari non è mai contemporaneo alla proprio punizione, né tampoco può corrispondere ad essa. Nel testo non abbiamo nessun appiglio per dire che si possa credere mentula moechatur corrispondente o assimilabile alla punizione prevista per tale azione. L'ingegnosa spiegazione per ipsa olera olla legit potrebbe valere se non fosse rapportata a mentula moechatur attraverso hoc est, che difficilmente può esprimere un rapporto di causa-effetto o, eventualmente, di preconizzazione, che invece è quanto ci aspetteremmo in presenza di un'azione e relativa punizione.

Crediamo che si possa sollevare, con Baehrens, il dubbio se il testo di Catull. 94, così com'è stampato e interpretato comunemente, sia genuino. Non già per la lettura di Catull. 113 sopra ipotizzata, ma alla luce delle esegesi correnti di Catull. 94 la soluzione di Baehrens sembra meritare più attenzione di quanto non ne abbia avuta ${ }^{77}$.

La pars destruens di Baehrens sembra soddisfacente, mentre la pars construens è convincente solo per la menzione della lezione di $\mathrm{O}$ e per l'osservazione della paretimologia ${ }^{78}$ olla - olera che avrebbe un forte pendant con la paronomasia/paretimologia generata da Moecia oppure Moechula a contatto con moechatur. Purtroppo non appare cogente la ricostruzione:

tagonisti (cf. Catull. 57,6-9: morbosi pariter, gemelli utrique, / uno in lecticulo erudituli ambo, $[\ldots]$ / riuales socii puellularum).

${ }^{74}$ Claes, "Catullus c. 94", 66: "With a masculine subject the verb moechari means "to have a mistress, to be a seducer'; with a feminine subject it means 'to be a mistress, to be seduced'»."

${ }^{75}$ Damschen, "Catullus c. 94", 176.

${ }^{76}$ Cf. Damschen, "Catullus c. 94", 175: «Da Mentula nun nach Catulls Darstellung in c. 94, 1 ein überführter Ehebrecher ist (Mentula moechatur), scheint das Sprichwort des Pentameters auf die römischen Sanktionen für dieses Verbrechen hinzuweisen: olla pro ano (scil. Mentulae), olera pro raphanis».

${ }_{77}$ Già nella postuma edizione catulliana di Baehrens, curata nel 1893 da K.P. Schulze, Moechula non figura nemmeno in apparato.

${ }^{78}$ Etimologia, che è proprio il caso di definire varroniana: ab olla olera dicta, quorum fa gerere cruda olera (Var. ling. 5.108.6). 
«Mentula moechatur (et quid aliud ab 'ista diffututa mentula' potest expectari?), moechatur etiam Moecia (nec in hanc, ut ipsum nomen indicat, aliud quidquam conuenit); quodsi reputatur a Mentula moechante didicisse Moeciam moechari (CXIII) apparet aut nusquam aut hic ualere illud 'i. o. o. l'. sed, ut dixi, certius de hac re iudicare liceret, si de sensu prouerbii omnes essent exemptae dubitationes ${ }^{79}$. Rimandata la valutazione sulla connessione con Catull. 113, è la connessione delle due proposizioni chiasticamente legate, e quindi il nesso tra i due personaggi, che non convince.

Infatti Mentula moechatur moechatur Moechula costituisce un chiasmo in cui gli estremi variano determinando una giustapposizione di contenuti non del tutto equipollenti. Infatti da una parte Mentula moechatur registra, grazie alla sua anfibologia (nome comune / nome proprio), un'ovvietà, dato che, salvo casi particolari, una mentula è necessariamente presente nelle fornicazioni, dall'altra moechatur Moechula, col sostantivo in posizione enfatica, informa che l'oggetto della fornicazione, una moechula, è diventato il soggetto, ricoprendo il ruolo di un(a) Mentula.

Ecco la diversità dei due giochi di parole ed ecco, a nostro parere, la ragione dello "stress" sull'ordo uerborum in moechatur Moechula rispetto a Mentula moechatur. Con le prodezze di Mucia si raggiunge quel paradosso che quelle di Mamurra non possono raggiungere. A confronto si può citare Catull. 100.1-4, ove si ricorre alla citazione di un'espressione popolare per confermare una situazione: Caelius Aufillenum et Quintius Aufillenam / flos Veronensum depereunt iuuenum, / hic fratrem, ille sororem. hoc est, quod dicitur, illud / fraternum uere dulce sodalicium. Con una movenza simile a hoc est quod dicunt, non solo il poeta conferma un fatto singolare con una frase fatta, ma anche si concede un gioco di parole. Se si mantenesse mentula anziché Moechula si ottunderebbe il potenziale di lusus verbale tra v. 1 e 2 del carme 94 .

Tale esegesi potrà sembrare un poco lambiccata, ma sembra necessario trovare una compiuta rispondenza con ipsa olera olla legit. Infatti Baehrens aveva rinunciato a spiegare il contesto di tale frase, certamente per prudenza, ma anche, crediamo, perché la sua soluzione si basava più su Catull. 113 che non sulla frase proverbiale. Quindi ipsa olera olla legit si addice solo apparentemente alla frase Mentula moechatur, mentre, a ben vedere, compiuta è la somiglianza del proverbio con moechatur Moechula: come l'olla da sola ruba ciò che gli è paretimologicamente congenere, così Moechula compie un'azione che le è affine a livello etimologico.

Pertanto, se Mentula e olla possono condividere solo il fatto d'essere entrambi strumenti, invece l'affinità tra Moechula e l'olla è nell'immagine del detto, in quanto il contenitore non riceve gli olera, ma lui stesso li ruba e, parimenti, Mucia è adultera non perché sedotta da un qualsivoglia Mentula,

${ }^{79}$ Baehrens, Catulli Veronensis liber, volumen alterum, 576. 
ma perché se lo procura essa stessa. Si noti che, all'interno di una società maschilista, il paragone tra mentula e olla può essere antitetico, mentre molto più diretto, absit iniuria uerbis, quello tra una moechula e un'olla ${ }^{80}$.

Infatti se si volesse ammettere che all'olla corrisponde Mentula, olera legere in che rapporto starebbe col moechari di Mamurra? Mentula, lo ribadiamo più chiaramente, è anche una mentula: può il suo moechari essere confrontato con un'olla che olera legit? Infatti proseguendo con l'identificazione di Mamurra con l'olla si dovrà allora concordare col paradosso del "penetrated penis» di Claes ${ }^{81}$.

Oppure si dovrà rivalutare la proposta di Damschen, che vede nell'espressione sentenziosa del v. 2, un'allusione al fatto che il moechari di Mamurra

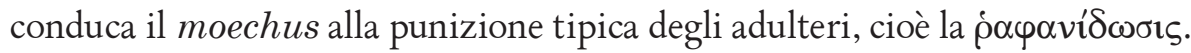

Quindi, ammessa e approfondita la bizzarra corrispondenza tra Mentula e l'olla che non solo Claes propone, ma anche il resto della critica, più o meno implicitamente accoglie ${ }^{82}$, si è costretti a seguire una delle declinazioni del "pene penetrato", di difficile, se non impossibile, dimostrazione (almeno finora). Infatti, a costo di dare l'impressione di ricorrere a una petizione di principio, escludo che Mamurra, qui evocato quale Mentula, possa assurgere al ruolo di fornicatore passivo: a meno che si possa dimostrare che a un omosessuale passivo si addica o sia testimoniato un soprannome che lo assimili a un (o al proprio) pene. Inoltre non si dimentichi Catull. 115.8 non homo, sed uero mentula magna minax, ove, se mai ce ne fosse stato bisogno, la "virilità" di Mamurra è esaltata con movenze epiche.

Una volta mostrata la plausibilità dell'emendamento di Baehrens, sia alla luce delle poche indicazioni di Catull. 94 (ciò̀ vagliatolo attraverso il proverbio) sia rispetto ad altre proposte esegetiche, sembra possibile continuare sulla scorta della pars construens dello studioso. Prima di tutto è interessante il dato codicologico: metula di $\mathrm{O}$, in luogo del secondo mentula, non è un argomento decisivo, perché se è possibile che $\mathrm{O}$ possa conservare una corruttela, più vicina a Moechula e destinata a essere normalizzata in mentula sul modello dell'incipit mentula moechatur, è altrettanto verisimile che metula possa essere un lapsus calami per mentula, determinato dalla mancata notazione del compendio della nasale da parte dell'antigrafo di $\mathrm{O}$ o di $\mathrm{O}$ stesso. Cionondimeno va rilevato che, senza eliminare del tutto la possibilità del lapsus, la presenza di mentula moechatur fa sì che moechatur mentula sia facilior rispetto a qualcosa che interrompe la simmetria chiastica del sin-

${ }^{80}$ A sostegno cf. i passi citati da V. Buchheit, "Ludicra latina”, Hermes 90, 1962, 254-5 e Damschen, “Catullus c. 94", 170-1.

${ }^{81}$ Cf. supra n. 70.

${ }^{82}$ Infatti, tutto sommato, anche nell'esegesi di Damschen, Mamurra viene penetrato analmente (seppur per punizione) e anche chi non prende in considerazione l'esegesi sessuale, essendoci di mezzo il moechari di un uomo chiamato Mentula, l'assimilazione di esso con un'olla finisce per rincondurci al paradosso di Claes. 
tagma. Inoltre si noti che metula è attestato anche in Burney $133^{83}$, che in Harl. $2574^{84}$ si legge mencula al posto del secondo mentula e che, a partire da Par. lat. $8458^{85}$, è attestato anche mens tua, sempre in luogo del secondo mentula (così anche Calfurnio e la prima edizione delle Emendationes di Avanzi ${ }^{86}$. Queste dissimilazioni, alcune probabili emendamenti, altre più probabili mende, lasciano aperta la possibilità che il dato codicologico possa supportare l'emendamento di Baehrens o, comunque, l'assenza di ripetizione: infatti la possibilità che tali letture siano tentativi di correzione conferisce loro un valore diagnostico, o quanto meno documentario: visto che né Calfurnio né Avanzi sembrano essere mossi da afflati eufemistici, il loro intervento segnala che già alla fine del XV secolo si nutrivano dubbi sulla bontà del solo secondo mentula. ${ }^{87}$

In secondo luogo Baehrens ha giustamente osservato che, come si è già avuto modo di dire, la paretimologia di ipsa olera olla legit trova un pendant in moechatur Moechula, sintagma che, a differenza di moechatur mentula, non presenta solo una trama parafonica, ma è una vera e propria figura etimologica.

Infine è necessario affrontare il problema della struttura sintattica del distico. Questo aspetto, come detto, è già stato oggetto di studio, tanto che, Baehrens a parte, sembra aver costituito finora l'unico problema riguardante la costituzione del testo del carme.

Pertanto è fondamentale esaminare il rapporto tra le proposizioni Mentula moechatur e moechatur Moechula. La prima, se intesa con Mentula=Mamurra, non costituisce una novità, vista la fama del luogotenente cesariano, né, tanto meno, se si coglie il senso letterale di mentula, volendone così sfruttare il potenziale anfibologico. Pertanto la prima proposizione può costituire o una constatazione di un dato di fatto oppure una domanda retorica. La seconda proposizione, moechatur Moechula, pone sullo stesso piano le "gesta" adulterine di un uomo e di una matrona, in quanto denotate dal medesimo verbo, in contrasto con la morale romana. Tuttavia la stessa proposizione, contemporaneamente, sfiora la tautologia, a causa della figura etimologica.

${ }^{83}$ London, British Library, Burney 133. Secondo M. Zicari, Scritti catulliani, a cura di P. Parroni, Urbino 1978, 167, il manoscritto non sarebbe imparentato con O. Vale la pena ricordare che i manoscritti imparentati con Burney 133 (sempre secondo Zicari, Scritti, 54), London, British Library, Egerton 3027 e Oliv. 1167, compendiano il secondo mentula.

${ }^{84}$ London, British Library, Harl. 2574 il quale apparterrebbe a una famiglia di manoscritti in cui "caratteri propri all'Oxoniensis s'incrociano con caratteri della famiglia del Sangermanensis» (Zicari, Scritti, 77). Per i rapporti di Harl. 2574 cf. Zicari, Scritti, 79-104.

${ }^{85}$ Paris, Bibliothèque nationale, Par. lat. 8458.

${ }^{86}$ Gli umanisti leggono mens tua solo in luogo del secondo mentula, addirittura il manoscritto parigino sostituisce entrambi i mentula.

${ }^{87}$ Quindi le incertezze della tradizione manoscritta seriore e le letture di alcuni umanisti si assommano alla lezione di O: naturalmente se non avessimo $\mathrm{O}$ queste osservazioni perderebbero di importanza 
Moechatur Moechula sembra costituire una declinazione particolare di un gioco di parole, basato sul duplice significato di Moechula, che, proprio come Mentula, è nome proprio e nome comune. Tuttavia, diversamente da Mentula, uno dei due significati confligge eticamente con moechatur, come si è detto.

Per comprendere l'esatto rapporto tra Mentula moechatur e moechatur Moechula può essere utile notare che il poeta deve inserire immediatamente una conferma, costituita da certe, poi una riprova data dalla conformità, di quanto va significando, a un detto d'uso comune (cf. hoc est quod dicunt).

Sembra più probabile che certe debba essere separato da quanto lo precede (Mentula moechatur moechatur Moechula): infatti una forte adesione, quale quella sintattica, dell'avverbio alla seconda parte del chiasmo sbilancerebbe la perfetta struttura. Inoltre non sembra nemmeno necessario che l'avverbio sia strettamente connesso sintatticamente, in contre-rejet, a hoc est quod dicunt. Infatti la frase può costituire un'ulteriore spiegazione alla conferma dell'avverbio.

La conferma significata da certe si spiega o con una precedente domanda $\mathrm{o}$, comunque, con un contenuto che necessitava di una ratifica ${ }^{88}$. Come già osservato, quanto viene confermato sembra il solo moechatur Moechula, poiché il detto ipsa olera olla legit si addice perfettamente unicamente alla seconda parte del chiasmo.

La critica, come detto, si divide tra chi vede nel chiasmo l'asindeto di due affermazioni o di un'affermazione seguita da una domanda. Se si parte dal vulgato mentula moechatur moechatur mentula, crediamo che l'andamento chiastico, comportando il passaggio da un ordine SV a VS, possa suggerire una variazione di intonazione, se non di punteggiatura, all'interno della ripetizione del medesimo sintagma mentula moechatur e che pertanto la puntuazione più probabile ${ }^{89}$ sia Mentula moechatur. Moechatur mentula? Certe etc. Infatti tale chiasmo, costruito da quattro elementi, ma due soli significanti, comporterebbe il passaggio in posizione di rema di mentula: è su questo sostantivo che la seconda frase e la figura retorica intera porrebbero enfasi, che, grazie a certe, andrebbe intesa quale richiesta di conferma dell'unica frase ripetuta invertendo l'ordine delle parole.

Tuttavia se il chiasmo comporta la giustapposizione di due proposizioni che condividono il verbo, ma non il soggetto, sembra difficile credere che l'enfasi posta sul secondo soggetto in posizione di rema implichi una richiesta

${ }^{88}$ Certe ha la stessa funzione in Catullo anche in cernitis, innuptae, iuuenes? consurgite contra; / nimirum Oetaeos ostendit Noctifer ignes. I sic certest (62.6-8) e nescio quid certest: an uere susurrat / grandia te medii tenta uorare uiri? / sic certest (80.5-7). Confrontabili con Catull. 62 le due occorrenze di certe in Catull. 80.5 e 7 e, ancora, in Catull. 30.7 certe introduce la sezione che segue le iniziali domande (retoriche).

${ }^{89}$ Potrebbe andare anche Mentula moechatur? Moechatur mentula,certe! Tuttavia, come già detto, così si perderebbe la perfezione del chiasmo. 
di conferma. Infatti la posizione enfatica può costituire una variazione di tono o più semplicemente marcare il cambiamento del soggetto a fronte della ripetizione del verbo. Ossia che senso ha chiedersi se moechatur Moechula, una volta detto che Mentula moechatur? Ancora, meno probabile sarebbe credere che moechatur Moechula costituisca la risposta a Mentula moechatur: infatti un tale rapporto tra le due frasi porrebbe l'attenzione sulla prima, che sarebbe una domanda circa un fatto in realtà assodato, quando invece sia il chiasmo sia il contenuto pongono enfasi sulla seconda frase.

Crediamo che l'enfasi sia data proprio dal gusto di giustapporre la stessa azione di due differenti personaggi, i cui nomi mostrano una particolare, ma diversa, ed è questo il punto, attitudine nei confronti dell'azione stessa. Il moechari del virilissimo Mamurra è giustapposto a quello della matrona Mucia, non già perché Mentula fosse sodomizzato (vuoi per scelta vuoi per punizione), ma perché il moechari di Moechula è etimologicamente ominoso come quello dell'olla che raccoglie gli olera. Inoltre è già stato ipotizzato per il personaggio femminile di Catull. 113 «eine aktive sexuelle Rolle»90x che appunto qui nel 94 sembra ricomparire. Da notare che tale ruolo è stato ipotizzato prescindendo dall'esistenza di un ciclo di Mucia- $\mu$ or $\chi$ ń e, naturalmente dalla proposta di Baehrens di emendare il secondo mentula in Moecula $=$ Mucia .

Poiché sembrano poche le possibilità che il chiasmo implichi la presenza di un'affermazione e di una domanda (o viceversa), la figura retorica può essere confrontata con l'afflato calligrafico di alcuni graffiti. Si veda, e.g., quisquis amat ualeat pereat qui nescit amare (CLE 946.1), ove il chiasmo si giustifica anche quale enfatizzazione del capovolgimento di situazione dalla prima alla seconda frase, e amat qui scribet pedicatur qui leget / qui osculat prurit, paticus est qui praeterit (CLE 45.1-2), ove la giustapposizione di beffe avviene prima con un parallelismo e poi attraverso due strutture chiastiche (solo la prima delle quali è perfetta). Naturalmente i testi citati presentano una combinazione di elementi popolari e dotti ${ }^{11}$. Quindi è difficile separare

${ }^{90}$ Hartz, Catulls Epigramme, 165. Si noti che lo studioso concorda con chi legge Maeciliam (Catulls Epigramme, 162).

${ }^{91}$ Si pensi anche alla reciprocità di influenza dei codici letterari e epigrafici in $C L E$. In particolare, "Catullo è un autore largamente tenuto presente nella tradizione dei $C L E$ e contemporaneamente è uno dei poeti che hanno mostrato maggior sensibilità nel recepire il codice epigrafico perfettamente contestualizzandolo» (P. Cugusi, "Ricezione del codice epigrafico e interazione tra carmi epigrafici e letteratura latina nelle età repubblicana e augustea", in $\mathrm{P}$. Kruschwitz (ed.), Die metrischen Inschriften der römischen Republik, Berlin 2007, 27. Del resto i carmi scoptici di Catullo possono presentare un punto di contatto con taluni testi di $C L E$, poiché sembrano essere stati concepiti con lo stesso intento di quei componimenti che rientrano nei Versus populares (cf. infra n. 92), cioè di «divulgare i difetti di un personaggio, fare dell'ironia sui suoi vizi, rendere il privato di dominio pubblico» (G. Cupaiuolo, Tra poesia e politica. Le Pasquinate nell'antica Roma, Napoli 1993, 13). In questo modo il verso destinatario del messaggio è la massa, per la quale tale produzione poetica svolge prima di tutto una 
ciò che interessa il lato culto del testo da ciò che attiene, più genericamente, alla funzione poetica del linguaggio. Forse Catullo sta utlizzando un registro linguistico che imita il composito stile dei graffiti e quindi il chiasmo può giustificarsi quale calligrafismo del graffito o, più in generale, dei uersus populares $^{92}$.

Pertanto due diverse interpunzioni sembrano possibili: 1) Mentula moechatur moechatur Moechula. Certe: hoc est quod dicunt etc., 2) "Mentula moechatur, moechatur Moechula"? Certe: hoc est quod dicunt etc. Così la frase, degna di un muro di Roma o di Pompei, può essere intesa (1) quale enunciazione affermativa che il poeta intende porre al riparo da eventuali obiezioni fornendo una spiegazione, oppure (2) quale enunciazione ${ }^{93}$ che il poeta intende porre, retoricamente, in dubbio. Altre occorrenze di certe in Catullo lasciano credere che debba essere preceduto da una domanda ${ }^{94}$. Così crediamo più probabile "Mentula moechatur, moechatur Moechula"? Certe: hoc est quod dicunt, "ipsa olera olla legit".

Crediamo che le difficoltà connesse all'identificazione di meciliam di $\mathrm{G}$ (mecilia di O R plerique recc.) per Catull. 113 e che le aporie segnalate da Baehrens in Catull. 94 possano essere affrontate con le letture proposte che, peraltro, si rafforzano l'una con l'altra.

I carmi 113 e 94 così letti implicherebbero l'esistenza di un piccolo ciclo di Mucia. Entrambi i testi presenterebbero diverse declinazioni di un medesimo gioco di parole costruito a partire dal nome della donna. Il denominatore comune è $\mu$ orx entrambi i testi si rivela e sprigiona il suo potenziale in una sententia finale. Il carme 113, con la sua elaborata cauillatio, rivelerebbe l'aition del soprannome di Mucia: quasi in una sorta di antomasia la donna guadagna l'appellativo per le sue altolocate relazioni adulterine e per le conseguenze che da esse discendono. Ancora, con l'esegesi proposta di Moechiliam, la sententia finale fecundum semen adulterio del v. 4 riceverebbe una luce particolare ${ }^{95}$,

funzione informativa, favorita da $\pi \alpha \rho \rho \eta$ í e, spessissimo, da un'oscenità non gratuita, bensì funzionale a denunciare la degenerazione del coté privato che viene reso pubblico (cf. Cupaiuolo, Tra poesia e politica, ibid.)

${ }^{92} \mathrm{Si}$ pensi il ricorso estenuato a strutture chiastiche settenari trocaici contro Cesare tramandati da Suet. Iul. 49.4 (Versus triumphales 1 Courtney = Versus populares in Caesarem 2 Bl.) Gallias Caesar subegit, Nicomedes Caesarem. I ecce Caesar nunc triumphat, qui subegit Gallias: / Nicomedes non triumphat, qui subegit Caesarem. Si noti anche il ricorso alla paronomasia nel parallelismo nunc triumphat (v. 3) non triumphat (v. 4), confrontabile con l'ipotizzato Mentula...Moechula.

${ }_{93}^{93}$ Indipendentemente da come si decida di interpungere il chiasmo può essere un lusus verbale che il poeta sottopone al lettore oppure la citazione di un detto già noto, la cui esistenza potrebbe essere anche solo fittizia.

${ }^{94} \mathrm{Cf}$. supra n. 88 con particolare riferimento ai passi in cui certe ha la funzione di ratificare quanto lo precede.

${ }^{95}$ A proposito si noti che c'è chi riscontra una certa debolezza nell'attacco al personaggio femminile di Catull. 113, proprio a causa del verso finale (Hartz, Catulls Epigramme, 166): con 
dovuta proprio a Mucia, il cui nome avrebbe in sé il seme dell'adulterio, poiché Mucilla evoca una figura mille volte moecha.

Il carme 94 accosta Mucia a un altro grande bersaglio di Catullo: Mamurra. Se le prodezze del Formiano sono note, quasi connaturate al suo soprannome, quelle della (ex)moglie di Pompeo sono apparentemente stupefacenti, poiché riconducibili a una sententia popolare. Si noti come il chiasmo Mentula moechatur, moechatur Moechula sia fortemente iconico: i due estremi convergono nel moechatur, quasi a dire che i due personaggi e le loro ipostasi (mentula e moecha) sono accomunati da e in questa azione. Tale oscena anfibologia trova un conforto nell'immagine del detto: l'atto dell'olla che prende gli olera sembra la traduzione in metafora agreste/vegetale dell'incontro moecha-mentula.

Mi si conceda il "priapismo" esegetico, ma, in questo caso, crediamo che la chiave di lettura del carme, con simili protagonisti non può che essere questa ${ }^{96}$. Anche in questo caso, oltre al paignion, cè un contenuto politico. L'invettiva politica è più marcata rispetto al carme 113. Infatti nella misura minima di questo distico, quasi da slogan o da graffito, non trova spazio il complesso lusus della cauillatio nominis di Catull. 113, non a caso indirizzato a Cinna. Così il contenuto priapistico si tramuta in metafora di malversazione politica. Dietro i due protagonisti del carme 94, si celano Cesare e Pompeo: Mucia, in quanto terza moglie di Pompeo e amante di Cesare, e Mamurra, in quanto diffututa mentula di Cesare e Pompeo (cf. Catull. 29.13 ss.).

All'immagine maschile della mentula che mangia tutto sembra che Catullo abbia affiancato quella di una adultera, anzi dell'adultera per eccellenza, scaturigine degli adulteri tra 70 e 55 a.C. Se è detto esplicitamente che Mamurra è lo strumento di distruzione di suocero e genero (cf. Catull. 29), meno diretto è il riferimento a Cesare e Pompeo dietro a Mucia, ma l'immagine è sempre la stessa. Ancora, se Mamurra è lo strumento per eccellenza di Cesare, in quanto appartenente alla sua fazione, allora Mucia è lo strumento di Pompeo, in quanto sua (ex)moglie ${ }^{97}$. I due personaggi sono simbolo, quasi ipostasi

questa lettura la sententia generale trova una callida declinazione particolare.

${ }^{96}$ Già Buchheit, "Ludicra latina", 255, sulla scorta della traduzione proposta e motivata da O. Weinreich, Die Distichen des Catull, Tübingen 1926, 22-5 «Wie sollt auch nicht, wie in Sprichwort es heißt: suchen die Scheide das Schwert?», insiste sull'esegesi oscena di tutto il testo: «apparet, quam facile etiam olla pro cunno adhiberi potuerit. Holus pro pene, quamquam exemplis certis probare non possumus, tamen initia multorum rei veneriae verborum a plantis arboribusque et earum fructibus orta esse in memoriam redigendum est». Tuttavia lo studioso, pur mostrando come mentula possa essere rappresentato da olus, non spiega come il proverbio e mentula moechatur possano andare insieme.

${ }^{97}$ Si noti come sia Mamurra sia Mucia abbiano collegamenti anche con Pompeo e Cesare: il Formiano ha militato anche sotto Pompeo (la praeda Pontica di Catull. 29.18 si riferisce ai guadagni di Mamurra durante la guerra mitridatica, vinta da Pompeo) e, naturalmente, Mucia è stata amante di Cesare. 
visti i soprannomi, della politica di malversazione dei due uomini di spicco della res publica del tempo.

Pertanto, questo eventuale ciclo di Mucia, risulterebbe analogo per contenuti e parallelo a quello di Mentula. Infatti il liber si presta a individuare carmi riconducibili a un denominatore comune e non di rado gli insiemi così ricavati sono interconnessi: ad esempio due sono i carmi indirizzati a Furio e ad Aurelio che si intersecano con i carmi dedicati a Lesbia, ma, allo stesso tempo, esistono anche carmi dedicati al solo Furio e al solo Aurelio. Allo stesso modo i carmi di Mucia presenterebbero alcuni temi del ciclo di Mamurra che però sarebbe evocato solo in uno dei due.

Qualora si accettasse tale lettura, sarebbe poi necessario affontare lo status di chilia (113.3: mil(l)ia V), grecismo inopinatamente congetturato da Giovanni Fosforo. L'intervento potrebbe essere rubricato quale fortunata e fortunosa congettura di un altrimenti, per quanto mi risulta, ignoto umanista. Oppure qualora volessimo sottovalutare le capacità di un erudito che professava in un ambiente vicino al mondo greco e intendessimo sopravvalutare i contatti del medesimo con l'Oriente, si potrebbe anche avanzare l'ipotesi che Fosforo avesse emendato non ope ingenii, ma ope codicum che circolavano in area orientale. In tal guisa, come abbiamo già detto, si ricadrebbe nel quarto punto del decalogo di Pasquali. Ma dal momento che il quarto punto è, se non indimostrabile, quanto meno materia che può essere affidata "soltanto a pochissimi tra i filologi» ${ }^{98}$, prudenza vuole che, nel caso di adesione alla lettura proposta, non si scoperchi alcun "vaso di Pandora" e si rubrichi chilia quale intervento umanistico inteso a fornire una patina di maggiore erudizione al testo, che già poteva incitare alla creazione di grecismi, vista la presenza di un moechilia / moechalia che veniva inteso quale plurale di un

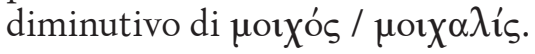

${ }^{98}$ Pasquali, Storia della tradizione, xvi. 
